\title{
$\bullet \bullet \bullet \bullet$ \\ Mehrsprachigkeit \\ und Multikulturalität \\ in original \\ deutschsprachigen und \\ ins Deutsche \\ übersetzten Comics \\ im Wandel der Zeit
}

ANNIKKI LIIMATAINEN 
In diesem Beitrag werden die Multikulturalität und die Mehrsprachigkeit in Comics untersucht. Unter Mehrsprachigkeit versteht man die Verwendung von zwei oder mehr Sprachen in demselben Text. Das Gesamtkorpus für diesen Beitrag besteht aus für unterschiedliche Zielgruppen publizierten älteren wie auch neueren Comics. Anhand einer Belegsammlung von ursprünglich deutschsprachigen und ins Deutsche übersetzten Comics soll gezeigt werden, welche Änderungen im Gebrauch von Wortgut aus anderen Sprachen im Laufe der Zeit festgestellt werden können, welche Funktionen die Mehrsprachigkeit in Comics hat, welches die sprachlichen Variationen und Kombinationen sind, die in Comics vorkommen, und was passiert, wenn diese ins Deutsche übersetzt werden. Im Mittelpunkt der Untersuchung steht darüber hinaus die Frage, wie die Mehrsprachigkeit der Ausgangskultur so übersetzt werden kann, dass die Mehrstimmigkeit beim Übersetzen nicht verloren geht, so dass man auch in der Zielkultur die Bedeutung der sprachlichen Variationen genau so verstehen kann wie in der Ausgangskultur.

Schlüsselwörter Mehrsprachigkeit, Multikulturalität, Code-Switching, Mehrstimmigkeit, Übersetzung von Comics, Deutsche Comics, Kommunikative Funktionen der Mehrsprachigkeit

\section{Gegenstand und Zielsetzung der Untersuchung}

Aus sprachwissenschaftlicher Sicht, wie auch aus dem Blickwinkel der Translationswissenschaft betrachtet, ist die Mehrsprachigkeit in Comics ein noch wenig untersuchtes Forschungsgebiet. Neben den Beiträgen von Mitrache (2004) zur Übersetzung von Phraseologismen lateinischen Ursprungs anhand der Comic-Serie Asterix und Liimatainen (2020) zur Mehrsprachigkeit und Multikulturalität in deutschen und finnischen Comics als Ausdruck des gesellschaflichen Wandels, den populärwissenschaftlichen Lexika zu lateinischen Zitaten in Asterix-Comicserie (z. B. Goscinny/Uderzo 2004; Valta 2013) sowie dem nicht-wissenschaftlichen Nachschlagewerk zu Fremdwörtern in den finnischsprachigen Übersetzungen der britischen Kriegscomic-Serie Commando (Vesterinen et al. 2007) gibt es meines Wissens keine weiteren Arbeiten zur Mehrsprachigkeit in Comics. Anhand der Untersuchung der 
Sprache des Comics, der zu den translationswissenschaftlich „weitgehend unerschlossenen Bereichen“ (Kaindl 2004: 326) gehört, gewinnt man einen Einblick in ein Genre der Belletristik, das eine breite und vielfältige Leserschaft hat. Die Comicforschung ist zurzeit ${ }^{1}$ stark im Wachstum begriffen, und die Untersuchung der Mehrsprachigkeit in Comics gewährt uns gleichzeitig auch Einblicke in den gesellschaftlichen Wandel.

Die Untersuchung der Mehrsprachigkeit ist wiederum wichtig in der heutigen globalisierten Welt - wie Busch (2013: 7) festgestellt hat - sowohl gesellschaftlich-politisch als auch wissenschaftlich betrachtet. Im Hinblick auf global agierende Unternehmen, weltweite wirtschaftliche Beziehungen und neue Raumkonfigurationen auf politischer Ebene, im Hinblick auf weitverbreitete Mobilität, Migration und Teilnahme an übernationalen Kommunikationsnetzwerken wird Mehrsprachigkeit immer häufiger als Teil der Alltagsrealität erkannt. Im Kontext der erwähnten Phänomene spielen die Herkunftskulturen und -sprachen eine besondere Rolle, dadurch dass sie zu neuen Formen der kulturellen und sprachlichen Heterogenität einen Beitrag leisten.

Die Entwicklung des Englischen zur Lingua franca im 20. Jahrhundert beeinflusste und beeinflusst bis heute die meisten Sprachen der Welt und damit auch das Deutsche. Anglizismen durchdringen heutzutage fast alle Lebensbereiche, von dem beruflichen Alltag über Wissenschaft, Politik, Massenmedien, Wirtschaft, Werbung, Informatik und Technik bis hin zu Freizeitaktivitäten, Sport, Tourismus, Kultur und Musik.

Mit der Entwicklung von grenzüberschreitenden Jugendkulturen tragen auch Jugendliche zur Verbreitung von Entlehnungen aus dem angloamerikanischen Raum bei (Neuland 2007: 22). Als Zeichen der Globalisierung können besonders jugendtypische Entlehnungen in vielen Ländern der Welt nachgewiesen werden. Im Sprachgebrauch Jugendlicher macht sich in jüngster Zeit auch der Einfluss von Migrantensprachen als Gebersprachen bemerk-

1 Comics waren im 20. Jahrhundert kaum Gegenstand wissenschaftlicher Forschung (Schwarz 2004, 676). Die Übersetzungswissenschaft hat ihr Interesse auf multimodale Texte erst ab den 1990er Jahren vermehrt gelenkt. In seinem seither immer wieder zitierten Aufsatz „Comics und Cartoons: (k)ein Gegenstand der Übersetzungswissenschaft?“ lenkte Peter A. Schmitt den Fokus auf die bis dahin kaum beachtete translatologische Relevanz dieser Textsorte (Schmitt 1997). Zum Forschungsstand s. u. a. Schwarz (2004: 676) und Kaindl (2010). 
bar. Nach dem bisherigen Wissen trifft dies in erster Linie auf Gruß- und Abschiedsformeln zu, z. B. das türkische hadi in der Mischform hadi tschüss und das italienische ciao in ciao bis dann! (Neuland 2007: 23). Neuland (ebd.: 22) hebt jedoch hervor, dass Jugendliche in Deutschland nicht besonders viele Fremdwörter in ihrem Sprachgebrauch benutzen, und macht vielmehr auf die unterschiedlichen Gebrauchshäufigkeiten von Entlehnungen in unterschiedlichen Sprachverwendungssituationen und Textsorten aufmerksam (s. auch Neuland/Schubert/Steffin 2007).

In zweisprachigen Sprachgemeinschaften werden die Sprachen häufig miteinander vermischt. Dies gilt beispielsweise auch für Finnland, wo es an der Süd- und Westküste viele alte Sprachgemeinschaften mit den beiden Landessprachen Finnlands, d. h. Finnisch und Schwedisch, gibt. Der momentane Gebrauch der anderen Sprache ist besonders typisch im Sprachgebrauch der schwedischsprachigen Jugendlichen, speziell in der Hauptstadt Helsinki (Saari 2007: 179-80).

Aus den erwähnten Gründen könnte vermutet werden, dass die Mehrsprachigkeit, insbesondere Entlehnungen aus dem Englischen, aber auch aus anderen Sprachen, auch in der Textsorte Comic nicht unüblich sind. Darüber hinaus wird hier angenommen, dass die Mehrsprachigkeit in Comics neueren Datums verstärkt bemerkbar ist.

Die Darlegungen in dem vorliegenden Beitrag basieren auf dem Studium unterschiedlicher Comics. Anhand einer Belegsammlung von ursprünglich deutschsprachigen wie auch ins Deutsche übersetzten Comics soll gezeigt werden, welche Änderungen im Gebrauch von Wortgut aus anderen Sprachen im Laufe der Zeit festgestellt werden können, welche Funktionen die Mehrsprachigkeit in Comics hat, welches die sprachlichen Variationen und Kombinationen sind, die in Comics vorkommen und was passiert, wenn diese ins Deutsche übersetzt werden.

Nach der Einleitung werden in Kap. 2 zunächst die theoretischen Grundlagen und der aktuelle Forschungsstand im Hinblick auf die der Arbeit zugrunde liegenden Fragestellungen beschrieben. Kapitel 3 widmet sich der Vorstellung des Materials. In Kap. 4 wird die Mehrsprachigkeit in den herangezogenen Comics mit Rücksicht auf den Umfang und die Vorkommenshäufigkeit, die Herkunftssprachen und Erscheinungsformen, auf kommunikative Funktionen der fremdsprachlichen Ausdrücke sowie auf die Strategien 
und Verfahren, die für die Übersetzung von Mehrsprachigkeit verwendet worden sind, systematisch untersucht. In Kap. 5 werden die aus der Analyse gewonnenen Ergebnisse zusammengefasst.

\section{Mehrsprachigkeit und Übersetzen}

Ungeachtet der Tatsache, dass sowohl Mehrsprachigkeit als auch Übersetzen schon sehr lange existieren, hat die Mehrsprachigkeit in der Übersetzungswissenschaft bisher nur eine marginale Erscheinung dargestellt, und erst in den letzten Zeiten hat man begonnen, das Übersetzen von mehrsprachigen Texten ausführlicher zu untersuchen (s. z. B. Meylaerts 2010: 227; Nurmi 2013: 111). Aufgrund von Globalisierung, Migration und zunehmendem Sprachkontakt sowie der Tatsache, dass Mehrsprachigkeit zu einem zentralen Forschungsthema beispielsweise in der Soziolinguistik, Sprachlehrforschung und Fremdsprachendidaktik geworden ist, gewinnt die Untersuchung der Übersetzung von mehrsprachigen Texten auch in der Übersetzungswissenschaft immer mehr an Bedeutung. Die jüngsten Veröffentlichungen decken eine Vielzahl von Bereichen und Themen ab, z. B. literarische Übersetzung, audivisuelle Übersetzung, Lokalisierung, sowie eine breite Palette von geografischen und institutionellen Einstellungen, u. a. Australien, Südafrika, die EU (Meylaerts 2010: 227). Der Schwerpunkt der Untersuchungen, die sich mit literarischen Texten befassen, liegt hautsächlich im Vergleich zwischen dem Ausgangstext und der zielsprachlichen Übersetzung (Nurmi 2013: 112-113). Beim Übersetzen von Comics ist dieser Ansatz jedoch unzureichend, denn der Text stellt lediglich einen Teil des Ganzen im Comic dar: Das unveränderbare Bild legt den Text fest. Das Bild kann dem Übersetzer bei seiner Arbeit einerseits helfen, es kann beispielsweise neue Ideen für das Übersetzen von Wortspielen geben, andererseits kann es aber die Möglichkeiten des Übersetzers einschränken.

Während das Übersetzen traditionell als Kommunikation verstanden wird, bei der sowohl die Ausgangssprache als auch die Zielsprache inbegriffen sind, versteht man unter Mehrsprachigkeit die Verwendung von zwei oder mehr Sprachen in demselben Text oder in einer Übersetzung (Grutman 2009: 182). Um dem einsprachigen Leser den Text verständlicher zu machen, werden nach fremdsprachigen Abschnitten zum Text häufig auch In-Text-Übersetzun- 
gen hinzugefügt. Wenn also die Übersetzung auch innerhalb eines Textes und nicht nur zwischen Texten stattfindet, stellt die literarische Mehrsprachigkeit die Definition der traditionellen Übersetzung als Ersatz eines Textes in Sprache A durch einen anderen Text in Sprache B in Frage. (Meylaerts 2010: 227)

In mehrsprachigen Texten kommen die Sprachen im Allgemeinen jedoch nicht gleichberechtigt vor, sondern eine der Sprachen ist die Hauptsprache, und mit Hilfe der anderen Sprachen wird die Geschichte zum Beispiel in eine gewisse zeitliche, sprachliche oder kulturelle Umgebung integriert oder die Fremdheit bzw. die Verschiedenheit einer Person bzw. einer Personengruppe hervorgehoben.

Ein typisches Merkmal mehrsprachiger Sprecher ist es, dass sie in Gesprächen untereinander innerhalb des Gesprächs und manchmal sogar innerhalb eines Satzes die Sprache wechseln. In diesem Fall spricht man von CodeSwitching. Unter Code-Switching wird in der vorliegenden Untersuchung in Anlehnung an Riehl (2013: 385) „de[r] Wechsel zwischen zwei (oder mehr) Sprachen [...] innerhalb ein und derselben kommunikativen Interaktion [verstanden]. Der Wechsel kann sowohl einzelne Lexeme als auch einen ganzen Diskursabschnitt betreffen." Code-Switsching führt das Gespräch aber nicht auf die andere Sprache über, sondern ist stets in einem einzigen Redebeitrag eingeschlossen, und die Gesprächspartner verwenden dieselbe Hauptsprache auch weiterhin.

In der Forschung wird darüber diskutiert, ob von Code-Switching nur dann gesprochen werden kann, wenn es bei der anderssprachigen Äußerung um eine ganze Phrase oder einen Teilsatz geht, oder auch bereits dann, wenn nur ein Wort aus der anderen Sprache verwendet wird (Riehl 2013: 385). Im vorliegenden Beitrag werden in Anlehnung an Riehl (ebd.) auch einzelne Wörter zum Code-Switching gezählt, vorausgesetzt dass das Wort spontan verwendet wird und nicht bereits ein fester Bestandteil des Lexikons in der Varietät dieser Sprachgemeinschaft ist. Nicht selten steht die Code-Switching-Sprache in gewissem Verhältnis zu der Hauptsprache. Dieses Verhältnis ist vom jeweiligen Thema des Werkes abhängig und dadurch ein Teil des Themas und sollte aus diesem Grund auch in der Übersetzung beibehalten werden.

Beim Überwechseln von der Hauptsprache in eine andere Sprache beschränkt sich der Einfluss der anderen Sprache nicht nur auf die Übernahme von einzelnen Wörtern. Der Ausgangstext kann zusätzlich zu einzel- 
nen Wörtern fremden Ursprungs auch noch u. a. Phraseologismen, Fluchwörter, adressatenspezifische Invektiven (z. B. Du Schwein!; Le cochon!) sowie Song- und Schlagertexte beinhalten, die von einer anderen Sprache kommen als von der, die größtenteils den Ausgangstext ausmacht. (Vgl. auch Bastian 2013; Enell-Nilsson/Hjort 2013; Fiedler 2016a; Liimatainen 2011a, 2011b, 2013, 2015, 2020; Nurmi 2013; Nurminen 2013).

Ein Panel, d. h. ein Einzelbild eines Comics, kann außer den Erzählertexten (in Kästchen) sowie Sprech- und Denkblasen auch Detailtexte beinhalten, die entscheidend zum Beispiel zur Vermittlung von Lokal- und Zeitkolorit der Geschichte beitragen oder den Geschichten Authentizität verleihen. Detailtexte oder Inserttexte bzw. Inserts (Fiedler 2016b: 35) sind Texte z. B. auf Verkehrszeichen, Plakaten, Ladenschildern, Gebäudeaufschriften oder in Zeitungen, die Teile der Umgebung sind, die im Comicbild zu sehen ist. Detailtexte sind beispielsweise Toponyme, deren Hauptfunktion ist, das vorgegebene Territorium, in dem die Handlung stattfindet, zu bestimmen und unter anderem durch Straßen- und Gebäudenamen das Milieu detailliert zu beschreiben.

Zu den wichtigsten Aufgaben des Übersetzers gehört zu entscheiden, wie er mit Kulturspezifika oder mit solchen Erscheinungen, die auch der Ausgangskultur fremd sind, umgeht, beispielsweise mit der Mehrsprachigkeit des Comics. Die Comicfiguren sprechen eine Sprache, die für sie charakteristisch ist, können aber in ihrer Rede Wörter auch aus einer anderen Sprache verwenden. Der Übersetzer muss klären, welche Kulturspezifika er beibehält, um die spezielle Atmosphäre des Comics nicht zu zerstören und ihm nicht sein Zeit- und Lokalkolorit zu nehmen. Dabei soll der Übersetzer auch noch die semiotische Komplexität der Textsorte Comic berücksichtigen, d. h. die Interaktion zwischen visuellem und verbalem Code, die Beteiligung sowohl von Sprache und Bild an der Konstituierung von Gesamtbedeutung (vgl. Schmitt 2003: 266). Sind die verbalen und nonverbalen Konstituenten in der Übersetzung nicht aufeinander abgestimmt, verliert der Comic seine Kohärenz und infolgedessen möglicherweise seine Akzeptabilität als Text (ebd.).

Neben den weiteren übersetzungsrelevanten Eigenschaften von Comics (Schwarz 2004: 676) stellt das Übersetzen der Mehrsprachigkeit für den Übersetzer eine spezielle Herausforderung dar. Jede Sprachkombination im Originaltext ist speziell, und die sprachliche Situation der Zielkultur kann in Bezug auf die Situation der Ausgangskultur sehr unterschiedlich sein (Grutman 2006: 
22). Darüber hinaus sind die Lesegewohnheiten kulturspezifisch. Besonders problematisch ist das Übersetzen in dem Fall, wenn irgendeine von den im Ausgangstext verwendeten Fremdsprachen für die meisten Leser der Zielsprache völlig unbekannt ist. Auf Schwierigkeiten stößt der Übersetzer aber auch dann, wenn die im Ausgangstext verwendete Fremdsprache gleichzeitig die Zielsprache ist, d. h. die Sprache, in die das Werk übersetzt werden soll. In diesen Fällen besteht die Gefahr, dass die sprachlichen Elemente, die im Original die Andersartigkeit signalisieren, ihre indexikalische Bedeutung verfälschen und als vertraute Zeichen der Gleichheit gelesen werden. (Ebd. 22-23).

Das einfachste Verfahren, das der Übersetzer anwenden kann, ist, die Hauptsprache in die Zielsprache (ZS) zu übersetzen und die in den Ausgangstext eingebetteten Fremdsprachen wie im Original zu übernehmen (Diaz Cintas 2011: 220). Textteile in anderen Sprachen, die für das inhaltliche Verständnis des ZS-Lesers nicht so relevant sind, werden nicht übersetzt. Somit bleiben diejenigen Effekte, die beim Original mit der Mehrsprachigkeit erzielt werden, ebenfalls in der Übersetzung erhalten. Wird der fremdsprachige Bestandteil in der Übersetzung in derselben Form beibehalten wie im Ausgangstext, ist es üblich, dass die Übersetzung beispielsweise in der Fußnote gegeben oder beim Text eine Erklärung hinzugefügt wird, so dass auch solche Leser, die nur die Zielsprache beherrschen, den gesamten Text verstehen, ohne dass dessen Mehrstimmigkeit verloren geht. Das zweite Verfahren ist die einsprachige Übersetzung (vgl. Diaz Cintas 2011: 221), die sprachliche Unterschiede tilgt, da die Ausgangssprache, sowohl die Hauptsprache als auch die weiteren Sprachen, komplett durch die Zielsprache ersetzt wird. Ein Vorteil davon ist, dass der ZS-Leser keine Verständnisschwierigkeiten hat. Damit geht aber der Effekt der sprachlichen Vielfalt verloren. Verloren geht auch der symbolische Wert der Mehrsprachigkeit, die in bestimmten Situationen gezielt verwendet wird. (Ebd.)

Im Mittelpunkt der Überlegungen dieser Untersuchung steht die Frage, wie die Mehrsprachigkeit der Ausgangskultur so übersetzt werden kann, dass man auch in der Zielkultur die Bedeutung der sprachlichen Variationen genau so verstehen kann wie in der Ausgangskultur. Sprachwechsel und sprachliche Variationen haben im Werk stets einen bestimmten Zweck, und wird dieser Zweck in der Übersetzung ausgelassen oder nicht berücksichtigt, so hat dies einen Einfluss beispielsweise auf die Charakterisierung der Perso- 
nen des Werkes als Vertreter ihrer soziokulturellen Umgebung, des Weiteren auf die Beschreibung ihrer Beziehungen untereinander und schließlich auf die Gesamtbedeutung des Werkes.

\section{Materialgrundlage}

Im Folgenden soll anhand von Beispielen aus mehreren älteren und neueren Comics, von denen ein Teil original deutschsprachige und ein Teil ins Deutsche übersetzte Comics sind, gezeigt werden, welche Änderungen im Gebrauch vom Wortgut aus anderen Sprachen im Laufe der Zeit festgestellt werden können. Darüber hinaus soll aufgezeigt werden, welche Gebersprachen dabei dominant sind, welche die sprachlichen Variationen und Kombinationen sind, die in untersuchten Comics vorkommen, und was passiert, wenn diese ins Deutsche übersetzt werden. Im Mittelpunkt des Interesses steht auch noch, welche Funktionen die Mehrsprachigkeit in Comics hat.

Da das Ziel der Untersuchung eine möglichst reiche und umfassende Deskription der Mehrsprachigkeit und Multikulturalität in Comics ist, schien es angebracht, einen eher qualitativen Forschungsansatz zu wählen. Um ein möglichst repräsentatives und vielseitiges Korpus zusammenzustellen, wurden für unterschiedliche Zielgruppen publizierte Comics von den 1970er Jahren bis zur Gegenwart gewählt, die unterschiedliche Themen behandeln. Insgesamt umfasst das Korpus 2614 Seiten. Neben humoristischen Zeitungsstrips und Werken mit Unterhaltungsfunktion bezeugen die in der jüngeren Vergangenheit entstandenen Comics (z. B. das Holocaust-Comic Maus von Spiegelman (1998), Tietäväinens Unsichtbare Hände aus dem Jahre 2014 sowie das autobiografische Comic Persepolis von Satrapi (2019)) deutlich, dass Comics - wie bereits Fiedler (2016b: 31) in ihren Untersuchungen festgestellt hat - „auch schwierige Themen bewältigen und dabei zu beachtlicher erzählerischer Tiefe und Komplexität gelangen können“.

Das Korpus ist untergliedert in zwei Subkorpora, wobei das Subkorpus 1 aus sieben original deutschsprachigen Comics besteht, während das Subkorpus 2 sechzehn Comics umfasst, die aus diversen Sprachen ins Deutsche übersetzt worden sind. Hinsichtlich der Form werden sowohl Comicstrips als auch Comic-Hefte, Comic-Alben und Graphic Novels berücksichtigt. 
Als Quelle der Untersuchung zur Mehrsprachigkeit dienen

in 1) original deutschsprachigen Comics (insgesamt 749 Seiten)

- Birbad, Bd. 1: Das Germanendorf von Donner und Blasco (1986),

- Der bewegte Mann von König (1987),

- Das Geheimnis der Lindenstraße. Erster Teil: Dresslers letzte Fahrt von Hinricher et al. (1994),

- Dr. Bubi Livingston. Du! Du! Du! ICH! ICH! ICH! von Kiefersauer (2000),

- Kinderland von Mawil (2017),

- Hat man erst angefangen zu reden, kann alles Mögliche dabei herauskommen von Beeler (2018),

- MOSAIK-Sammelband 19 (2019)

sowie in 2) deutscher Übersetzung

a) aus dem Französischen (insgesamt 642 Seiten)

- Die Schlümpfe 6: Rotschlümpfchen und Schlumpfkäppchen von Peyo und Delporte (1983),

- Asterix bei den Briten von Goscinny/Uderzo (1985),

- Ein außergewöhnliches Abenteuer von Boule und Bill: Bill ist fort! von Duchateau et al. (1991),

- Tim und Struppi: Die Krabbe mit den goldenen Scheren von Hergé (1998),

- Der Sohn des Asterix von Uderzo (2017, erste Veröffentlichung 1983),

- Lucky Luke: Das gelobte Land von Achdé/Jul (2018),

- Persepolis von Satrapi (2019),

b) aus dem Englischen (insgesamt 597 Seiten)

- Familie Feuerstein 3: Jagd auf den Steinhüpfer von Hanna und Barbera (1974),

- Woody Woodpecker. Neue lustige COMIC-Abenteuer von WOODY und seinen Freunden. Comic-Album Nr. 1 (1977),

- Aristocats. Die schönsten Disney-Geschichten 10 (1980),

- Donald Duck 2 von Barks (1994),

- Maus I und II von Spiegelman (1998a, 1998b),

- Mumins: Die gesammelten Comic-Strips von Jansson, Bd. 3 (2015), 
c) aus dem Finnischen (insgesamt 450 Seiten)

- Unsichtbare Hände von Tietäväinen (2014),

- COMIC ATLAS FINNLAND herausgegeben von Hommer und Hakkola (2014) sowie

d) aus dem Japanischen (176 Seiten)

- Another 2nd Period von Ayatsuji und Kiyohara (2017).

\subsection{Original deutschsprachige Comics}

Bis zum Anfang der 1980er Jahre blieben die deutschen Comics nahezu gänzlich auf den Kindersektor beschränkt. Seit den späten 1980er Jahren kam auch in Deutschland eine Kultur der anarchischen Comics auf. Ein wichtiger Vertreter dieser Kultur ist Ralf König mit seinem Werk Der bewegte Mann. Seit Mitte der 2000er Jahre gibt es immer mehr auch solche Comiczeichner, die ihre Werke in erster Linie über ihre Webseiten präsentieren, wie beispielsweise Kiefersauer mit Dr. Bubi Livingston. Du! Du! Du! ICH! ICH! ICH!. Die Comic-Kultur in der DDR war nicht so vielfältig wie in Westdeutschland, aber fast generell hochwertig mit MOSAIK als Flaggschiff. Zu den jüngeren Repräsentanten der deutschen Comic-Szene gehört u. a. der Berliner Mawil mit Kinderland. Der deutschsprachige Comic-Markt ist aber nicht so groß und absatzstark wie der in den meisten anderen europäischen Ländern.

Die Birbad Germanencomics von Donner und Blasco erschienen Mitte der 1980er Jahre. Das wohl von der Comic-Serie Asterix beeinflusste humorvolle regionale Comic, das im Eigenenverlag erschien, verbindet Elemente derselben (germanisches Dorf Anfang der Völkerwanderungszeit wehrt sich gegen die Hunnen) mit Fantasy-Elementen der germanischen Göttersagen. (Zeisberger o. J.)

Der Comicroman Der bewegte Mann von König erschien 1987. Mit einer Gesamtauflage von fast sieben Millionen Exemplaren ist König heutzutage der weltweit populärste Autor mit Nachdruck „schwuler Geschichten“. Seine Bücher sind bis jetzt in 15 Sprachen übersetzt worden. Im Carlsen-Verlag erschien in den Jahren 1992 bis 1995 die dreiteilige Comic-Reihe Das Geheimnis der Lindenstraße von Hinricher et al. Der humoristische Comicstrip Dr. 
Bubi Livingston² von Kiefersauer erscheint seit 1990 als Wochenendbeilage in der Westdeutsche Allgemeine Zeitung (WAZ).

In Kinderland setzt sich Mawil (2017) mit dem Leben von Kindern und Jugendlichen in Berlin in der DDR auseinander, mit einer Kindheit zwischen Jungen Pionieren und Kirche am Vorabend der Wende. Zum 25. Jahrestag des Mauerfalls legt Mawil mit Kinderland eine ebenso in die Tiefe gehende wie unterhaltsame Geschichte vor, die aufgrund persönlicher Erinnerungen und sorgfältiger Recherche ein lebhaftes Bild der letzten Tage der DDR zeichnet.

Der Schweizer Illustrator Beeler hat seine erste Graphic Novel Hat man erst angefangen zu reden, kann alles Mögliche dabei herauskommen 2018 herausgegeben, welche die Geschichte einer psychisch kranken Frau und ihres Sohnes erzählt. Die Frau, eine Schweizerin, hat sich ein neues Leben in einem kurdischen Dorf im Südosten der Türkei aufgebaut und versucht, ihre schwierige Vergangenheit hinter sich zu lassen. Die Comic-Zeitschrift MOSAIK wurde 1955 in Ost-Berlin gegründet. MOSAIK kann nicht nur auf eine lange Erscheinungsdauer zurückblicken, sondern ist gegenwärtig auch einer der ältesten und auflagenstärksten Comics deutscher Produktion. Seit 1976 reisen im MOSAIK die Abrafaxe auf abenteuerliche Weise durch die Zeiten und Regionen der Erde. MOSAIK wird heutzutage in viele Sprachen übersetzt. Die in Sammelband 19 abgedruckten Abrafaxe-Abenteuer erschienen erstmals 1982 in den MOSAIK-Heften 1 bis 4 .

Bis auf die Zeitschrift MOSAIK und teilweise auch das Comic-Magazin Fix und Foxi, das mit Unterbrechungen zwischen 1953 und 2010 herausgegeben wurde, beruhte die sehr geringe heimische Produktion in Deutschland hauptsächlich auf Übersetzungen aus dem frankobelgischen sowie amerikanischen Sprachraum (vgl. Meloni 2016, 160), die beide selbst eine vergleichsweise große eigene Comic-Tradition besitzen. Insbesondere Frankreich und Belgien sind Comic-Großmächte (Römpötti 2017, B5), und die französische Sprache ist die wichtigste Sprache in der Cartoon-Welt (Thuren 2018, 2). 


\subsection{Deutsche Übersetzungen von Comics}

Wie in 3.1. bereits erwähnt, beschränkt sich der deutsche Comic bis Anfang der 1980er Jahre nahezu völlig auf Kindercomics. Für den größsten Teil des 20. Jahrhunderts nach dem Zweiten Weltkrieg war der deutschsprachige Comicmarkt von übersetzten Comics wie The Adventures of Tintin (deutsch: Tim und Struppi), Asterix sowie Micky Maus geprägt. Gegen Ende des 20. Jahrhunderts begannen Superhelden und Manga eine große Präsenz auf dem Comic-Markt für übersetzte Ausgaben zu haben.

\subsubsection{Deutsche Übersetzungen von francobelgischen Comics}

Der Begriff francobelgischer Comic ist ein Kollektivum für Comics, die im französischen Sprachraum in Europa, hauptsächlich in Frankreich und Belgien, erstmals veröffentlicht werden. Frankreich und Belgien verfügen über eine lange Comic-Tradition, die gezeichneten Bildergeschichten werden dort vor allem als eigenständige Kunstform angenommen und erfolgreiche Comicpublikationen erreichen hohe Auflagen. Insbesondere in der Nachkriegsära war die Comicproduktion des französischsprachigen Europas qualitativ dominierend. Viele Comics in den Jahrzehnten nach dem Zweiten Weltkrieg schlugen einen komödiantischen Ton an und richteten sich hauptsächlich an ein jugendliches Zielpublikum. Aus dieser Zeit stammen auch die in Deutschland bekannte Comics Tim und Struppi, Asterix und Lucky Luke.

Die Schlümpfe (im französischsprachigen Original Les Schtroumpfs) wurden zunächst im französischen und niederländischen, ab Ende der 1960er Jahre auch im deutschen Sprachraum und in weiteren europäischen Ländern populär. Die Sprechweise der Schlümpfe, die im Schlumpfdorf im Verwunschenen Land leben, nennt man Schlumpfsprache, wobei es sich nicht um eine eigentliche fiktionale Sprache handelt, sondern nur um das Einfügen des Wortes Schlumpf anstelle des richtigen Wortes (z. B. Rotschlümpfchen oder Schlumpfkäppchen statt Rotkäppchen).

Die Asterix Comic-Serie enthält bis Ende des Jahres 2020 insgesamt 39 Bände. Asterix bei den Briten ist der achte Band der Comic-Reihe und erschien im Jahr 1966 auf Französisch und 1971 auf Deutsch. Der 27. Asterix-Band Der Sohn des Asterix erschien 1983 sowohl auf Französisch als auch in deutscher Übersetzung. Da die Comic-Serie Asterix auf eine lange Erscheinungsdauer 
vom Jahre 1961 bis heute zurückgeht, gestattet sie uns Einblicke in die Entwicklung der Sprache in übersetzten Comics.

Boule und Bill (im deutschen Sprachraum bekannt auch als Schnieff und Schnuff) ist eine belgische Comic-Reihe, die von den Erlebnissen eines siebenjährigen Jungen namens Boule und seines Hundes Bill handelt. Sämtliche Geschichten stammen aus dem alltaglichen Leben einer ganz gewöhnlichen Familie in einem ganz gewöhnlichen Wohnhaus und der direkten Umgebung. Die Krabbe mit den goldenen Scheren ist der neunte Band der Abenteuer von Tim und Struppi des belgischen Zeichners Hergé. Es ist der erste Band, in dem ein neuer Freund Tims, der trunksüchtige und ewig fluchende Kapitän Haddock, auftaucht, der Tim bei allen weiteren Abenteuern nicht mehr im Stich lassen wird. Diese vollständig überarbeitete Farbausgabe erschien erstmals 1943. Sowohl die Asterix- als auch die Tim und Struppi-Alben können zu den erfolgreichsten europäischen Comics gezählt werden und wurden bisher in mehr als 100 Sprachen übersetzt (Keromnes 2019).

Lucky Luke ist die Titelfigur einer seit 1947 erscheinenden belgischen Comic-Serie des Zeichners Morris. Lucky Luke ist der bekannteste und erfolgreichste Western-Comic weltweit. Das Album Das gelobte Land (französischer Originaltitel: La Terre promise) wurde bei seinem Erscheinen sowohl in Frankreich als auch in Deutschland mit großem Interesse aufgenommen. Das gelobte Land bezieht sich auch auf die Thematisierung der Einwanderung aschkenasischer Juden (mittel-, nord- und osteuropäische Juden und ihre Nachfahren) in die Vereinigten Staaten im 19. Jahrhundert.

Die autobiografische Geschichte Persepolis von Satrapi (2019) wurde bisher in 25 Sprachen übersetzt und ist mit über einer Million verkaufter Bücher weltweit eine der bekanntesten Graphic Novels. Die gebürtige Iranerin Satrapi hat ihre eigene Lebensgeschichte benutzt, um die Geschichte ihrer Heimat, die Auseinandersetzung mit der Politik und Lebensweise im Iran, zu erzählen. (Tabeling 2007) In einer einfachen Bildsprache berichtet Satrapi von der islamischen Revolution im Jahr 1979, dem Leben im Iran danach, von ihrem Exil in Wien sowie der Rückkehr in ihr Heimatland. 


\subsubsection{Deutsche Übersetzungen von englischsprachigen Comics}

Wie in 3.1. bereits festgestellt wurde, verfügt neben dem frankobelgischen Sprachraum auch der amerikanische Sprachraum über eine relativ große eigene Comic-Tradition.

Als Erica Fuchs 1951 die ersten amerikanischen Walt-Disney-Stories zu übersetzen anfing, hatte die Textsorte Comic, in der Alltagsgeschichten oder abenteuerliche Reisen humorvoll erzählt werden, in Deutschland noch keine Tradition. Fuchs, die fast 40 Jahre die amerikanischen Geschichten eindeutschte, adaptierte sie für den deutschsprachigen Rezipienten, passte sie an die deutsche Kultur an und wählte bei der Übersetzung der Originaltexte den Weg der freien Übersetzung. Bekannt wurde Fuchs insbesondere durch ihre Übersetzungen der Geschichten von Carl Barks rund um die Familie Duck. (Meloni 2016, 159-161)

Weder im Heft Familie Feuerstein von Hanna und Barbera (1974) noch im Comic-Album Woody Woodpecker und im Heft Aristocats, die 1977 und 1980 erschienen sind, wird mitgeteilt, von wem die Übersetzung stammt. Familie Feuerstein (englisch The Flintstones) spielt in der steinzeitlichen Stadt Steintal, deren Gesellschaft mit der amerikanischen Mittelschicht des 20. Jahrhunderts verglichen werden kann. In dieser Stadt leben eiszeitliche Tiere zusammen mit Höhlenmenschen, die jedoch über angepasste heutige Technik verfügen. Woody Woodpecker (im Deutschen früher auch Hacky der Specht) ist eine Comicfigur, die von Walter Lanz gestaltet wurde. Woody Woodpecker zählt zu seinen bekanntesten Cartoonfiguren. Zwischen 1947 und 1984 war Woody Woodpecker Hauptfigur in mehr als 200 Comic-Heften. Aristocats erzählt die liebenswerten Abenteuer einer Katzenfamilie aus reichem Hause in Paris des Jahres 1910.

Der Comic mit dem Titel Maus des amerikanischen Comicautors Art Spiegelman veränderte den Status von Comics gründlich. Maus wurde als Graphic Novel, als bebilderter Roman, benannt und sein Status als Kritikerliebling brachte unmittelbar ein breites Spektrum an neuen Graphic Novels hervor. (Petersen 2021) In Maus wird in Form einer Tierfabel die authentische Leidensgeschichte des Vaters des Autors, des polnischen Juden Wladek Spiegelman, berichtet, der Auschwitz überlebte. Eine zweite, parallele Erzählebene beschreibt den Kampf des Sohnes um die Liebe und Anerkennung seines gebrochenen, kranken und erbitterten Vaters. In Maus verbindet Spiegelman 
die Autobiografie mit der psychologischen Analyse, die Erzählung mit der historischen Dokumentation. Spiegelman brauchte 13 Jahre, um die Geschichte seiner Famile zu vervollständigen. Er reiste auch nach Auschwitz, um mehr darüber zu erfahren. ${ }^{3}$ (Bacconini/Zanettin 2014: 105).

Die Mumins, der erfolgsreichste finnische Comic, sind von der finnlandschwedischen Schriftstellerin Tove Jansson erfundene Trollwesen. Zwischen 1954 und 1975 wurde der Mumin-Comicstrip in Drei-Bild-Streifen täglich in der englischen Zeitung Evening News veröffentlicht. Dieser Zeitungscomic war an Erwachsene adressiert (Tolvanen 2015) und wurde bald von ca. 120 Zeitungen in über 40 Ländern auf der ganzen Welt abgedruckt. Die Mumin-Comics erschienen in den 1950er und 1960er Jahren auch in vielen Tageszeitungen des deutschen Sprachraums, später auch in Jugendzeitschriften und Illustrierten. Anfang der 1980er Jahre brachte der Bastei-Verlag eine Heftreihe heraus, in der die Comics jedoch teilweise gekürzt und textlich verändert wurden.

\subsubsection{Deutsche Übersetzungen von finnischsprachigen Comics}

Auch finnische Comics werden auf der ganzen Welt geschätzt, und im Ausland sind ungefähr hundert finnische Cartoons veröffentlicht worden (Thuren 2018, 2).

Tietäväinens Näkymättömät kädet (deutsche Übersetzung des Titels Unsichtbare Hände) erschien 2011. Im Comic wird die Lebensrealität von Gastarbeitern im 21. Jahrhundert thematisiert: niedrige Bezahlung, schlechte Arbeitsbedingungen und die Hoffnung auf ein besseres Leben. Die Graphic Novel spielt in Spanien und erzählt die Geschichte der in Marokko arbeitslos gewordenen Hauptfigur, die Geschichte des Familienvaters Rashid. Um seine Familie weiterhin ernähren zu können, verlässt er diese, um in Europa Arbeit zu suchen. Er emigriert als illegaler Einwanderer ohne Pass nach Südspanien. Dort versucht er, sich ein neues Leben aufzubauen. Die dortigen Treibhausbesitzer haben großen Bedarf an Gastarbeitern und so wird Rashid einer von vielen. Unsichtbare Hände umfasst mehr als 200 Seiten verdichtet und verwandelt die auf Band aufgenommene Geschichte des Vaters in eine grafische Erzählung (vgl. Baccolini/Zanettin 2014: 128). 
und Tietäväinen arbeitete fünf Jahre daran. Zunächst las er für seinen Comic verschiedene Bücher, um sich genauer über die Situation der Menschen dort zu informieren. Danach reiste er zusammen mit einem Anthropologen noch nach Marokko und Spanien, um vor Ort weitere Nachforschungen anzustellen. In Unsichtbare Hände werden Fakten und Fiktion zusammengefügt.

Im Jahre 2014 war Finnland Ehrengast der Frankfurter Buchmesse und zu diesem Anlass hat Kalle Hakkola gemeinsam mit dem Hamburger ComicZeichner Sascha Hommer einen Comic Atlas Finnland auf Deutsch herausgegeben, der Comics von 12 zeitgenössischen finnischen Comic-Künstlern präsentiert, und zwar von Marko Turunen, Reetta Niemensivu, Tommi Musturi, Ville Ranta, Anna Sailamaa, Jarno Latva-Nikkola, Mika Lietzén, Jaakko Pallasvuo, Matti Hagelberg, Hanneriina Moisseinen, Amanda Vähämäki und Roope Eronen. Der Atlas, der 237 Seiten umfasst, ist der vierte Band in der Serie Finnish Comics Annual. Der Comic Atlas Finnland zeigt eine breit gefächerte Auswahl einer eigenwilligen europäischen Comickultur.

\subsubsection{Deutsche Übersetzungen von japanischen Comics}

Japans Comic-Industrie zählt zu den größten der Welt. Seit Mitte der 1980er Jahre finden die als Mangas bezeichneten Comics auch im Westen immer mehr Verbreitung. Unter Manga werden im westlichen Sprachgebrauch hauptsächlich Comics japanischen Ursprungs verstanden. Die Wurzeln des japanischen Mangas reichen bis in das japanischen Mittelalter zurück, seine heutige Form ist dagegen in hohem Grade durch die westlichen Einflüsse im 19. und 20. Jahrhundert geprägt (Ossmann 2004: 11-12). Auf den westlichen Comicmärkten werden japanische Mangas häufiger übersetzt als alle anderen fremdsprachigen Comics. Während der deutsche Comic-Markt bis in die 1990er Jahre von Übersetzungen aus dem Englischen und Französischen dominiert wurde, haben Übersetzungen aus dem Japanischen seitdem Vorrang vor Übersetzungen aus allen anderen Sprachen. (Jüngst 2014, 50) Der Durchbruch für Manga in Deutschland kam 1997 mit der 8000 Seiten umfassenden Serie Dragon Ball von Akira Toriyama (von Törne 2016).

Mangas wenden sich in erster Linie an die Zielgruppe der Leser von 10 bis 18 Jahren, die sowohl in Japan als auch in den westlichen Ländern die Hauptkonsumenten von Comics sind. Darüber hinaus sind aber auch noch 
Genres (z. B. Horror, Fantasy, Science Fiction, Comedy, Erotik, Sport, Krimi, Romantik, History) für andere Altersgruppen zugänglich (Ossmann 2004: 41, 45) Die außerhalb Japans bekannteste Form von Manga sind die sog. Story Mangas, die eine lange, häufig detailreiche Geschichte darstellen. Der japanische Manga bietet Raum für inhaltliche Tiefe und legt in erster Linie Wert auf die differenzierte Kennzeichnung der Stimmung einer Geschichte oder der Gedanken und Gefühle der Comicfiguren. Darauf ist zurückzuführen, dass japanische Comics Tausende von Seiten umfassen können. (Ossmann 2004: 28, 32-33) Mangas werden auf dem deutschsprachigen Markt fast ausschließlich in Taschenbuchform veröffentlicht. Mehrere Kapitel einer Serie werden zu einem Sammelband zusammengefasst, und die Bände umfassen ca. 200-230 Seiten. (Ossmann 2004: 17, 31).

\section{Mehrsprachigkeit in Comics}

Die herangezogenen Comics wurden auf Mehrsprachigkeit systematisch untersucht. Berücksichtigt wurden solche Wörter und längere Einheiten (u. a. Phraseologismen, Gebete und Schlagertexte) fremden Ursprungs, die im Text spontan verwendet werden und nicht bereits ein fester Bestandteil des Lexikons in der Varietät der deutschen Sprachgemeinschaft sind. Um die Forschungsfragen beantworten zu können, werden die Befunde in folgender Hinsicht ausgewertet:

- im Hinblick auf den Umfang und die Vorkommenshäufigkeit der fremdsprachigen Ausdrücke,

- im Hinblick auf die Herkunftssprache der Ausdrücke,

- im Hinblick auf die Erscheinungsform (einzelne Wörter, längere Einheiten) der Ausdrücke,

- im Hinblick auf kommunikative Funktionen der Mehrsprachigkeit.

\subsection{Umfang der Mehrsprachigkeit und Herkunftssprachen}

Der Zweck der folgenden Auswertungen ist es, festzustellen, wie hoch der Anteil der fremdsprachigen Ausdrücke in den original deutschsprachigen Comics sowie in den ins Deutsche übersetzten Comics ist und wie sich die Belege auf verschiedene Herkunftssprachen verteilen. 


\subsubsection{Umfang der Mehrsprachigkeit und Herkunftssprachen} in den original deutschsprachigen Comics

Was die ursprünglich deutschsprachigen Comics, nämlich Birbad: Das Germanendorf von Donner und Blasco (1986), Der bewegte Mann von König (1987), Das Geheimnis der Lindenstraße von Hinricher et al. (1994), Dr. Bubi Livingston Du! Du! Du! ICH! ICH! ICH! von Kiefersauer (2000), Kinderland von Mawil (2017), Hat man erst angefangen zu reden, kann alles Mögliche dabei herauskommen von Beeler (2018) sowie MOSAIK-Sammelband 19 (2019) betrifft, tritt eine nur relativ geringe Anzahl von insgesamt 63 fremdsprachigen Ausdrücken auf. Die quantitative Verteilung sowie die Herkunftssprachen der fremdsprachigen Ausdrücke in den ursprünglich deutschsprachigen Comics stellen sich wie folgt dar:

Tab. 1: Mehrsprachigkeit in den original deutschsprachigen Comics.

\begin{tabular}{|c|c|c|}
\hline Comic & Herkunftssprache & $\begin{array}{l}\text { Anzahl der fremd- } \\
\text { sprachigen Ausdrücke/ } \\
\text { Herkunftssprache }\end{array}$ \\
\hline Birbad: Das Germanendorf (1986) & Latein & 1 \\
\hline \multirow[t]{2}{*}{ Der bewegte Mann (1987) } & Englisch & 3 \\
\hline & Französisch & 1 \\
\hline \multirow{3}{*}{$\begin{array}{l}\text { Das Geheimnis der } \\
\text { Lindenstraße (1994) }\end{array}$} & Englisch & 16 \\
\hline & Italienisch & 1 \\
\hline & Spanisch & 1 \\
\hline \multirow[t]{2}{*}{ Dr. Bubi Livingston (2000) } & Englisch & 11 \\
\hline & Französisch & 1 \\
\hline \multirow[t]{4}{*}{ Kinderland (2017) } & Russisch & 4 \\
\hline & Französisch & 1 \\
\hline & Türkisch & 1 \\
\hline & Englisch & 11 \\
\hline \multirow{4}{*}{$\begin{array}{l}\text { Hat man erst angefangen zu } \\
\text { reden, kann alles Mögliche } \\
\text { dabei herauskommen (2018) }\end{array}$} & Englisch & 2 \\
\hline & Ungarisch & 1 \\
\hline & Türkisch & 2 \\
\hline & unbekannt & 2 \\
\hline \multirow[t]{3}{*}{ MOSAIK-Sammelband 19 (2019) } & Arabisch & 1 \\
\hline & Spanisch & 2 \\
\hline & Latein & 1 \\
\hline Insgesamt & & 63 \\
\hline
\end{tabular}


Bemerkenswert ist das fast totale Fehlen der Mehrsprachigkeit sowohl in zwei älteren als auch in zwei neueren Comics. An erster Stelle bei den Fremdsprachen erscheint das Englische mit 43 Belegen. Dies entspricht 68,3 Prozent von allen Belegen. Daneben kommen von den Fremdsprachen Russisch (4 Belege), Französisch, Spanisch und Türkisch (je 3 Belege), Latein (2 Belege) sowie Italienisch, Ungarisch und Arabisch mit je 1 Beleg vor (s. Tab. 1). In Hat man erst angefangen zu reden, kann alles Mögliche dabei herauskommen von Beeler (2018) tauchen zwei Fremdwörter auf, deren Herkunftssprache mir bisher unbekannt geblieben ist.

Nicht berücksichtigt werden in dieser Untersuchung Personen- und Figurennamen fremden Ursprungs (z. B. Omar ben Salaad, Antonius von Padua, Lucky Luke, Sitting Bull) sowie Onomatopoetika. Da die Comicfiguren selten eine Fremdsprache ohne Fehler und Mängel sprechen, sind auch solche Befunde berücksichtigt worden, in denen der Satz nicht korrekt gebildet ist oder ein Wort nicht korrekt ausgesprochen wird, wie beispielsweise in Poisson en Bratatouille (Dr. Bubi Livingston, Strip 12), How you like him? (Maus I, Spiegelman 1998a, 16) oder in So ejn Unglick! (Lucky Luke, Achdé/Jul 2018, 35).

\subsubsection{Umfang der Mehrsprachigkeit und Herkunftssprachen in den aus dem Französischen übersetzten Comics}

In den aus dem Französischen ins Deutsche übersetzten Comics (Die Schlümpfe: Rotschlümpfchen und Schlumpfkäppchen von Peyo und Delporte (1983), Asterix bei den Briten von Goscinny/Uderzo (1985), Ein außergewöhnliches Abenteuer von Boule und Bill von Duchateau et al. (1991), Tim und Struppi: Die Krabbe mit den goldenen Scheren von Hergé (1998), Der Sohn des Asterix von Uderzo (2017), Lucky Luke: Das gelobte Land von Achdé/Jul (2018) sowie Persepolis von Satrapi (2019)) lässt sich die Mehrsprachigkeit zum Teil kaum, zum größten Teil sogar in hohem Maße nachweisen (s. Tab. 2). 
Tab. 2: Mehrsprachigkeit in den aus dem Französischen übersetzten Comics.

\begin{tabular}{llc} 
Comic & Herkunftssprache & $\begin{array}{c}\text { Anzahl der fremd- } \\
\text { sprachigen Ausdrücke/ } \\
\text { Herkunftssprache }\end{array}$ \\
\hline $\begin{array}{l}\text { Die Schlümpfe: Rotschlümpfchen } \\
\text { und Schlumpfkäppchen (1983) }\end{array}$ & Latein & 2 \\
\hline Asterix bei den Briten (1985) & Latein & 1 \\
\hline Ein außergewöhnliches & Spanisch & 18 \\
Abenteuer von & Englisch & 7 \\
Boule und Bill (1991) & Latein & 30 \\
& Französisch & 1 \\
& künstliche Sprache & 3 \\
\hline Tim und Struppi: Die Krabbe mit & Arabisch & 1 \\
den goldenen Scheren (1998) & künstliche Sprache & 5 \\
Der Sohn des Asterix (2017) & Latein & 3 \\
\hline Lucky Luke: & Englisch & 23 \\
Das gelobte Land (2018) & Jiddisch & 24 \\
& Hebräisch & 4 \\
& unbekannt & 7 \\
\hline Persepolis (2019) & künstliche Sprache & 4 \\
\hline Insgesamt & Persisch & 16 \\
\hline & Französisch & 34 \\
\hline & Englisch & 9 \\
\hline & & 17 \\
\hline
\end{tabular}

Auffallend ist auf der einen Seite das fast totale Fehlen der Mehrsprachigkeit in zwei älteren aus dem Französischen ins Deutsche übersetzten Comics (Die Schlümpfe sowie Tim und Struppi), auf der anderen Seite kann Mehrsprachigkeit in den meisten Comics dagegen in verhältnismäßig hohem Umfang nachgewiesen werden. Der Band Lucky Luke: Das gelobte Land beinhaltet eine Fülle von Anspielungen auf das osteuropäische Judentum und die aschkenasische Kultur. Fast durch den ganzen Band sprechen die Mitglieder der jüdischen Familie Stern in einer Sprechweise, die vom Jiddischen beeinflusst ist und im Comictext durch Lautverschiebungen wie ibrig statt übrig oder ejn statt ein markiert wird: „Iberhaupt dirfen wir kejn Tier essn, das erlegt wurde mit ejner Foierwaffe." (Lucky Luke, Achdé/Jul 2018: 19). 
Aus Tabelle 2 ist ersichtlich, dass in den deutschen Übersetzungen fremdsprachige Ausdrücke aus mehreren Herkunftssprachen existieren. Während die Comicserie Asterix die lateinische Sprache bevorzugt, macht sich in jüngster Zeit der Einfluss von Migrantensprachen als Gebersprachen auch in Comics verstärkt bemerkbar. Als Beispiel kann die Graphic Novel Persepolis von Satrapi (2019) angeführt werden. An erster Stelle in den aus dem Französischen übersetzten Comics erscheint von den Fremdsprachen jedoch das Englische mit 71 Belegen. Dies entspricht ca. 34 Prozent von allen Belegen.

Neben den fremdsprachigen Wörtern und Äußerungen wird in Tab. 2 auch das Vorhandensein einer künstlichen Sprache mit 22 Belegen berücksichtigt. Unter künstlicher Sprache versteht Kaindl (2004: 276) eine Sprache, die in Comics mit Hilfe unterschiedlicher grammatischer, syntaktischer, morphologischer und phonologischer Mittel produziert wird, wie etwa die Zahlwörter trecieno doj, trecieno tres in einer Sprechblase im Donald Duck Taschenbuch Peli poikki 4491/22 (Aku Ankan taskukirja 2017: 107). Berücksichtigt wurden aufgrund dessen u. a. die Sätze und Ausdrücke, die in den Sprechblasen in Abbildungen 1, 2 und 3 vorkommen.

In Abb. 1 wird eine künstliche Sprache zur individualisierenden und typisierenden Figurencharakterisierung der hochzivilisierten Marsianer in Mumins verwendet, in Abb. 2 zur Charakterisierung der marokkanischen Barbaren in Tim und Struppi. Die zunächst feindseligen Cree-Indianer (s. Abb. 3) sprechen in Lucky Luke: Das gelobte Land (2018: 43-44) eine künstliche Sprache, in der sich häufig Benennungen für Speisen mit Bezug zum Judentum verbergen (z. B. Mulukheia ist ein arabisches Gemüsegericht, das wie Schakschuka und Msoki zur jüdischen Küche gehört). 


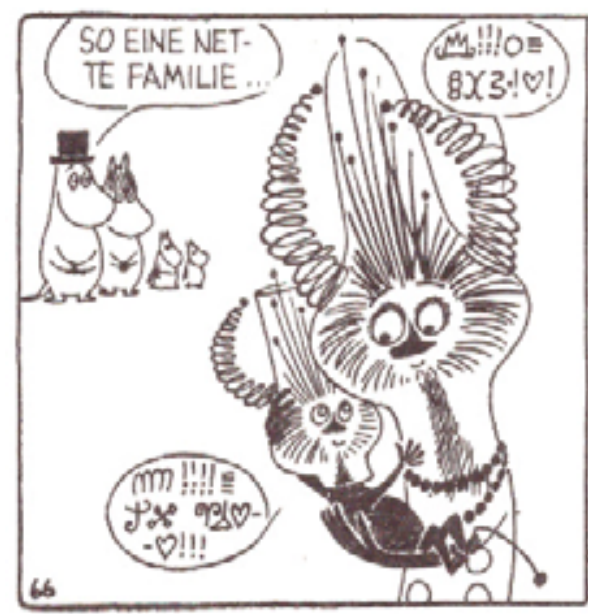

Abb. 1 Mumins (Jansson 2015: 57).

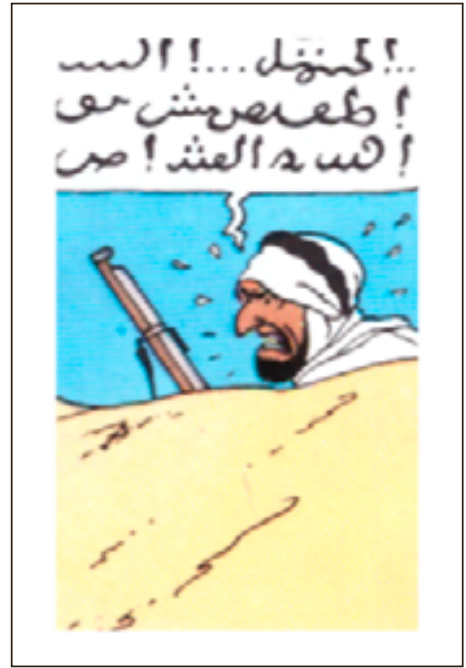

Abb. 2 Tim und Struppi: Die Krabbe mit den goldenen Scheren (Hergé 1998: 38).

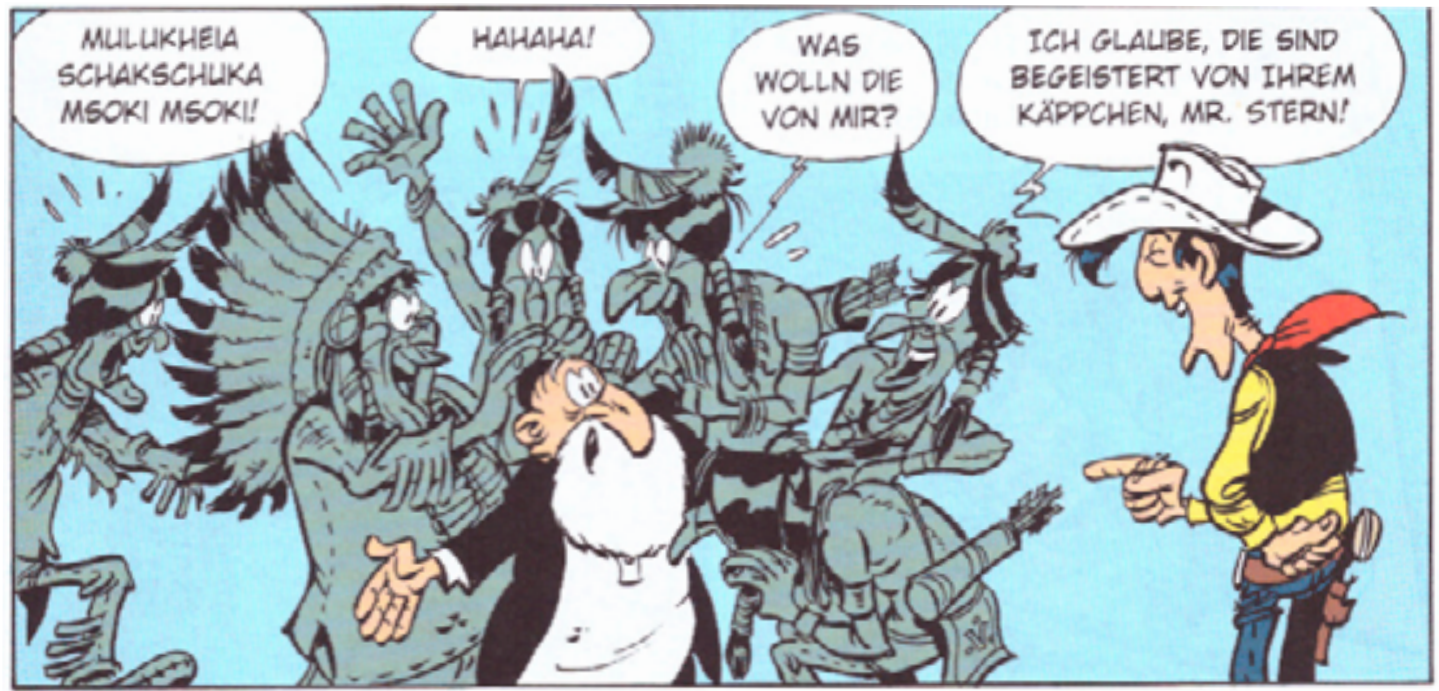

Abb. 3 Lucky Luke: Das gelobte Land (2018:43).

\subsubsection{Umfang der Mehrsprachigkeit und Herkunftssprachen} in den aus dem Englischen übersetzten Comics

Das dritte Subkorpus umfasst die aus dem Englischen übersetzten Comics Familie Feuerstein 3: Jagd auf den Steinhüpfer von Hanna und Barbera (1974), Woody Woodpecker. Neue lustige COMIC-Abenteuer von Woody und seinen Freunden. Comic-Album Nr. 1 (1977), Aristocats. Die schönsten DisneyGeschichten 10 (1980), Donald Duck 2 von Barks (1994), Maus I und II von Spiegelman: (1998a, 1998b) sowie Mumins: Die gesammelten Comic-Strips von 
Jansson, Bd. 3 (2015). Einen Überblick über die quantitative Verteilung der fremdsprachigen Ausdrücke sowie die Herkunftssprachen der fremden Ausdrücke in den aus dem Englischen übersetzten Comics gibt Tabelle 3:

Tab. 3: Mehrsprachigkeit in den aus dem Englischen übersetzten Comics.

\begin{tabular}{llc} 
Comic & Herkunftssprache & $\begin{array}{c}\text { Anzahl der fremd- } \\
\text { sprachigen Ausdrücke/ } \\
\text { Herkunftssprache }\end{array}$ \\
\hline Familie Feuerstein (1974) & keine & keine \\
\hline Woody Woodpecker (1977) & Englisch & 1 \\
\hline Aristocats (1980) & Spanisch & 1 \\
& Englisch & 1 \\
& Französisch & 5 \\
& Japanisch & 1 \\
\hline Donald Duck 2(1994) & Englisch & 15 \\
\hline Maus I und II (1998a, 1998b) & Englisch & 16 \\
& Hebräisch & 4 \\
& Polnisch & 9 \\
& Französisch & 4 \\
& Jiddisch & 5 \\
& unbekannt & 5 \\
\hline Mumins (2015) & Englisch & 4 \\
& künstliche Sprache & 6 \\
\hline Insgesamt & & 77 \\
\hline
\end{tabular}

Im Vergleich zu den aus dem Französischen übersetzten Comics tritt in dem aus dem Englischen übersetzten Korpus eine nur relativ geringe Anzahl von fremdsprachigen Ausdrücken auf. In vier der untersuchten Comics kommen keine oder nur einige wenige fremdsprachige Ausdrücke vor. Auch in diesem Subkorpus dominiert von den Herkunftssprachen das Englische mit 37 Belegen, was 48 Prozent von allen Belegen entspricht. An zweiter Stelle kommen Französisch und Polnisch mit je 9 Belegen. 

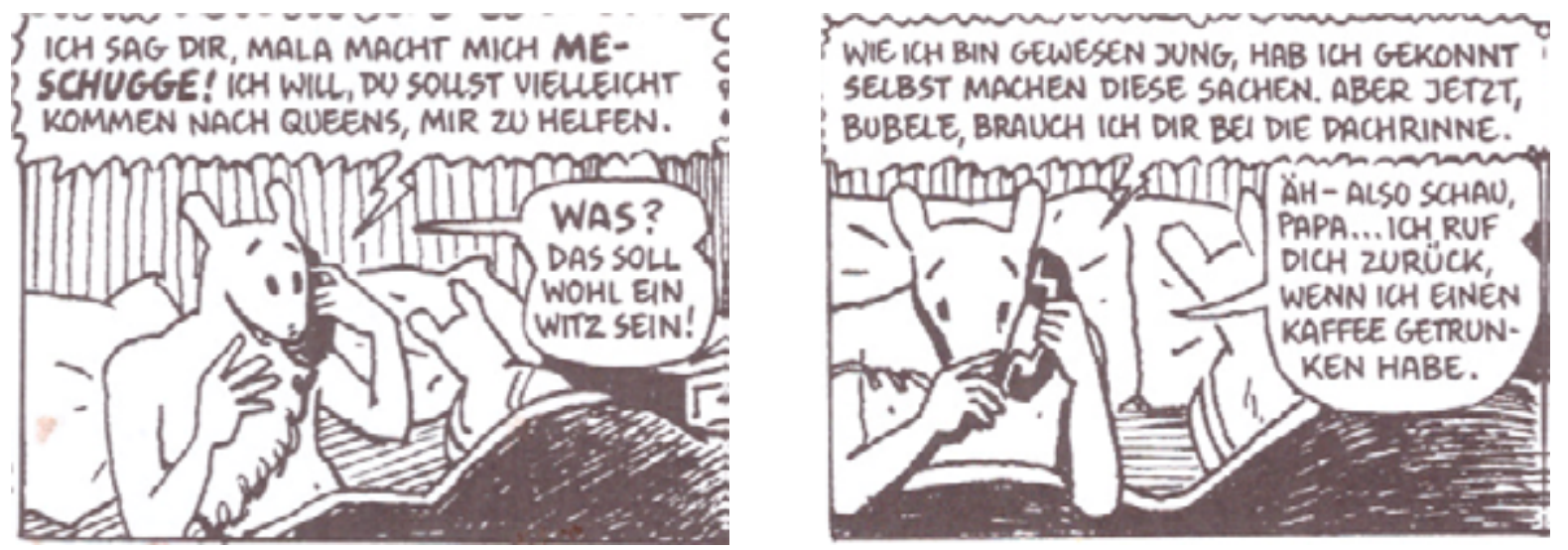

Abb. 4: Maus I (Spiegelman 1998a: 96).

In Maus I und II (Spiegelman 1998a, 1998b) spielt auch das Jiddische eine wichtige Rolle, denn der Protagonist Wladek Spiegelman hat Jiddisch als Muttersprache, verständigt sich aber je nach Gesprächsparter auch in zwei weiteren Sprachen, nämlich auf Polnisch und in gebrochenem Englisch. Daher haben die Übersetzer einen analogen Sprachstil gewählt, so dass der Protagonist in der deutschen Ausgabe „analog zum englischen Original ein von jiddischer Grammatik geprägtes Deutsch“ spricht (Vorbemerkung der Übersetzer in Spiegelman 1998b: 4). Wladeks Vokabular ist hauptsächlich das des amerikanischen Standard-Englisch, mit einer Reihe von jiddischen Wörtern (Abb. 4; s. auch Baccolini/Zanettin ${ }^{4}$ 2014: 125).

\subsubsection{Umfang der Mehrsprachigkeit und Herkunftssprachen in den aus dem Finnischen übersetzten Comics}

Das Subkorpus 4 umfasst das aus dem Finnischen übersetzte Album Unsichtbare Hände von Tietäväinen (2014) sowie den Sammelband COMIC ATLAS FINNLAND (2014). Tabelle 4 verdeutlicht, dass sich die Mehrsprachigkeit insbesondere in Unsichtbare Hände in hohem Maße nachweisen lässt (insg. 102 Belege), während in Comic Atlas Finnland fremdsprachige Ausdrücke viel seltener vorkommen (36 Belege).

4 Der Beitrag von Baccolini und Zanettin (2014) diskutiert die Übersetzungen von Maus in eine Reihe von Sprachen und Kulturen und untersucht, wie die Verwendung von gebrochenem Englisch in den Übersetzungen entnommen wird. 
Tab. 4: Mehrsprachigkeit in den aus dem Finnischen übersetzten Comics.

\begin{tabular}{llc} 
Comic & Herkunftssprache & $\begin{array}{c}\text { Anzahl der fremd- } \\
\text { sprachigen Ausdrücke/ } \\
\text { Herkunftssprache }\end{array}$ \\
\hline Unsichtbare Hände (2014) & Arabisch & 29 \\
& Spanisch & 43 \\
& Englisch & 16 \\
& Französisch & 2 \\
& Italienisch & 2 \\
& Finnisch & 4 \\
& Malagasy & 1 \\
& unbekannt & 5 \\
\hline Comic Atlas Finnland (2014) & Französisch & 8 \\
& Englisch & 9 \\
& Finnisch & 19 \\
\hline \multirow{2}{*}{ Insgesamt } & & 138 \\
\hline
\end{tabular}

An erster Stelle von den Fremdsprachen erscheint in diesem Korpus das Spanische mit 43 Belegen. Dies entspricht etwa 31 Prozent von allen Belegen. An zweiter Stelle kommt Arabish mit 29 Belegen, an dritter und vierter Stelle Englisch und Finnisch mit 25 bzw. 23 Belegen. In Unsichtbare Hände kommen auch noch fünf Bezeichnungen vor, deren Herkunftssprache mir bisher unbekannt geblieben ist. Zwei davon tauchen im Vorwort des Werkes auf:

Bittet man die alten Fischer aus den nördlichen Hafenstädten Marokkos, von dieser Meerenge zu erzählen, so berichten sie von den naghaze - launischen kleinen Wellen -, von unberechenbaren Strömungen sowie von Ostwinden, sharqi genannt, deren Wut jedes Schiff überrascht, das vom Atlantik her kommt. (Tietäväinen 2014: 4)

Die Bedeutung dieser Bezeichnungen stellt weder dem ausgangssprachlichen noch dem zielsprachlichen Leser Probleme dar, denn ihre Bedeutung wird sowohl im Ausgangs- als auch im Zieltext erklärt und dem Leser gleichzeitig ein Informationsangebot geliefert. 


\subsubsection{Umfang der Mehrsprachigkeit und Herkunftssprachen in dem aus dem Japanischen übersetzten Comic}

Die quantitative Verteilung der fremdsprachigen Ausdrücke sowie die Herkunftssprachen der fremden Ausdrücke in dem aus dem Japanischen ins Deutsche übersetzten Comic Another 2nd Period zeigt Tabelle 5.

Tab. 5: Mehrsprachigkeit in dem aus dem Japanischen übersetzten Comic.

\begin{tabular}{llc} 
Comic & Herkunftssprache & $\begin{array}{l}\text { Anzahl der fremd- } \\
\text { sprachigen Ausdrücke/ } \\
\text { Herkunftssprache }\end{array}$ \\
\hline Another 2nd Period (2017) & Japanisch & 7 \\
& Englisch & 2 \\
\hline Insgesamt & & 9 \\
\hline
\end{tabular}

In Japan legt man großen Wert auf Etikette und gute Umgangsformen, was sich auch an den japanischen Anreden zeigt, die ein wesentlicher Teil der japanischen Höflichkeitssprache sind. Die Wahl der richtigen Höflichkeitssilbe ist eine Frage des Respekts. In Another 2nd Period (Ayatsuji/Kiyohara 2017) kommen von diesen Silben -sun, -kun, -chan sowie -sensei vor. Darüber hinaus werden in der deutschen Ausgabe japanische Texte auf einem Handy-Display und von einem Schild an einer Innenwand der Schule sowie von einem Dokument unverändert übernommen, die aber durch deutsche Übersetzungen bzw. lateinische Buchstaben oder Nummern in der Fußsnote ergänzt werden, weil sie für den Handlungsverlauf wichtige Informationen vermitteln. Als Herkunftssprachen kommt mit zwei Belegen auch noch das Englische vor.

\subsubsection{Zusammenfassung}

Was die ursprünglich deutschsprachigen, die aus dem Englischen wie auch die aus dem Japanischen übersetzten Comics anbelangt, tritt in den meisten von diesen eine nur relativ niedrige Anzahl von fremdsprachigen Ausdrücken auf. Im Vergleich zu diesen drei Korpora lässt sich die Mehrsprachigkeit in den aus dem Finnischen und aus dem Französischen übersetzten Comics hauptsächlich in verhältnismäßig hohem Maße nachweisen. Anhand der 
untersuchten Korpora lässt sich beweisen, dass der Anteil vom Wortgut aus anderen Sprachen im Laufe der Zeit insbesondere in übersetzten Comics deutlich gestiegen ist.

In Comics, die hauptsächlich für Kinder und Jugendliche gedacht sind (z. B. Aristocats, Die Schlümpfe, MOSAIK, Woody Woodpecker, Tim und Struppi, Another 2nd Period), tritt Mehrsprachigkeit weniger oft auf als in Comics, die sich in erster Linie auf die Zielgruppe von Erwachsenen konzentrieren (z. B. Persepolis, Maus, Unsichtbare Hände). Dies dürfte darauf zurückzuführen sein, dass Mehrsprachigkeit gewisse Fremdsprachenkenntnisse voraussetzt und sie wird daher in erster Linie in Comics für erwachsene Leser verwendet.

Von den Herkunftssprachen erscheint an erster Stelle das Englische mit 178 Belegen, was 35,9 Prozent von allen Belegen (496) entspricht. Neben dem Englischen tauchen vor allem Spanisch (55 Belege), Latein (insbesondere dank der Comic-Serie Asterix mit 46 Belegen), Französisch und Persisch (je 34 Belege), Arabisch (33 Belege) sowie Finnisch (23 Belege) auf. Eine geringere Rolle als Herkunftssprache spielen Hebräisch, Polnisch, Japanisch, Russisch, Italienisch, Türkisch, Ungarisch, Malagasy und die künstlichen Sprachen. In Lucky Luke: Das gelobte Land (Achdé/Jul 2018) und Maus I und II von Spiegelman (1998a, 1998b) spielt die jiddische Sprache bei der Charakterisierung der Comicfiguren eine entscheidende Rolle. Wie nachgewiesen werden konnte, ist in jüngster Zeit der Einfluss von Migrantensprachen als Gebersprachen auch in Comics zunehmend zu erkennen.

\subsection{Sprachliche Realisation der Mehrsprachigkeit}

Den Belegen ist zu entnehmen, dass die Mehrsprachigkeit viele Erscheinungsformen haben kann. Im untersuchten Korpus kommen zusätzlich zu einzelnen Wörtern fremden Ursprungs auch noch fremdsprachige Interjektionen (z. B. Well, Bobby, an die Arbeit! (Boule und Bill (Duchateau et al. 1991: 42)); O lalá (Persepolis (Satrapi 2019: 259)), Phraseologismen, Fluchworte, adressatenspezifische Invektiven, d. h. Äußerungen von absichtlich beleidigendem Charakter, kurze Sätze (Fragesätze, Befehle), Gebete sowie Song- und Schlagertexte vor.

Die Analyse ergab, dass einzelne Wörter und Wortgruppen, die gelegentlich auch Hybridbildungen (s. Beisp. 2) sein können, den größten Teil der Belege 
fremden Ursprungs bilden und dass diese Ausdrücke aus vielen verschiedenen Herkunftssprachen stammen. Die Wörter und Wortgruppen treten in Sprechblasen, in Erzählertexten, Denkblasen und Inserts (s. Abb. 5 und 6) auf.

Zum einen wird für das Wort oder die Wortgruppe fremden Ursprungs weder im Text noch in der Fußnnote eine zielsprachige Entsprechung oder Erklärung gegeben. In den meisten Fällen handelt es sich um einfache Ausdrücke aus den Schulfremdsprachen, beispielsweise Englisch, Französisch, Spanisch oder Latein (Beispiele 1-4), wobei vom Zieltextrezipienten keine allzu großen Fremdsprachenkenntnisse erwartet werden. Es gibt aber auch solche Ausdrücke, die für die meisten Leser der Zielsprache völlig unbekannt sind (s. Beispiele 5 und 6).

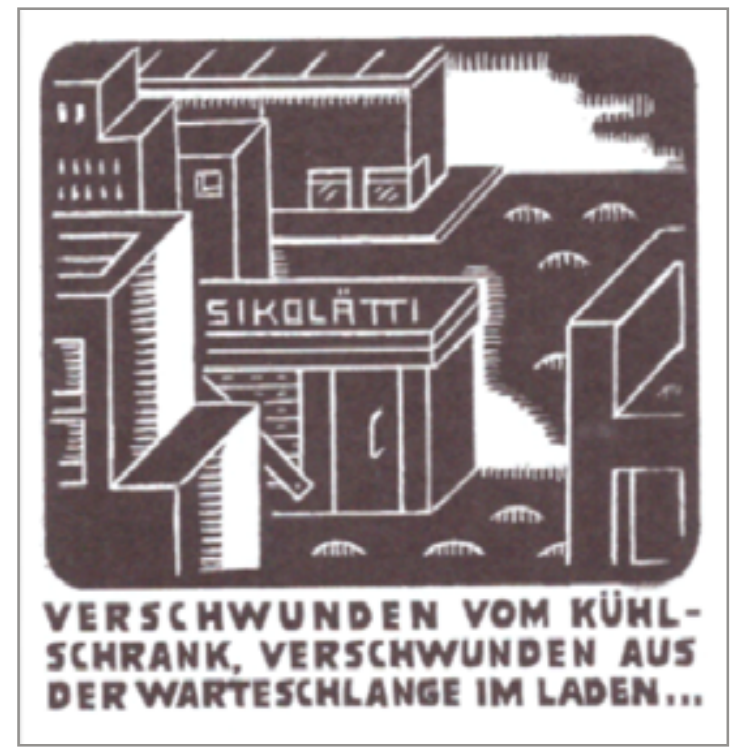

Abb 5: Comic Atlas Finnland (Hommer/Hakkola 2014: 167).

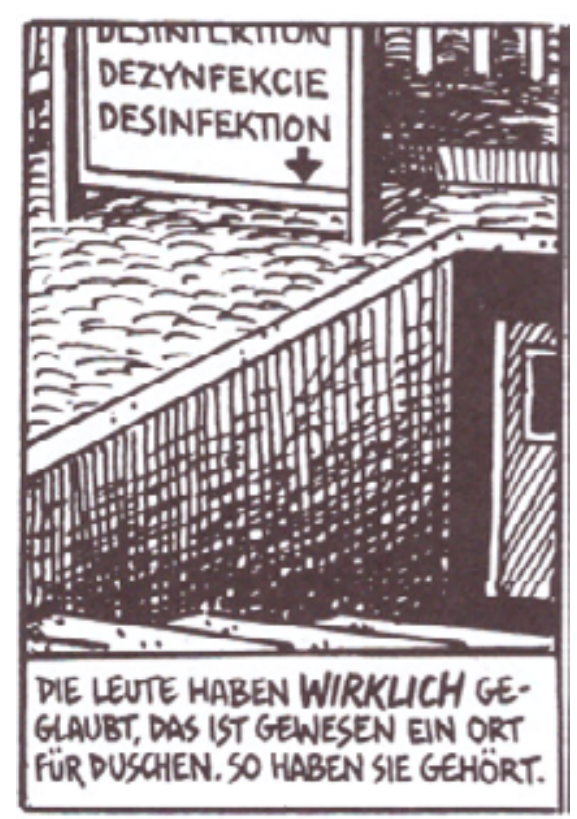

Abb. 6: Maus II

(Spiegelman 1998b: 70).

1. Und was hat's gebracht? Nothing.

(Der bewegte Mann, König 1987: 110)

2. Coming Out Probleme, Subkultur, Anmache...

(Der bewegte Mann, König 1987: 11)

3. brravo! una paella grrrande! FÜRRR DIE GANZE

FAMILIE! no?! (Boule und Bill, Duchateau et al. 1991: 2) 
4. Hunnen Ante Portas! (Birbad: Das Germanendorf, Donner und Blasco 1986: 48)

5. Das stimmt... man müsste schon Nike-Logos auf die Djellabas ${ }^{5}$ nähen, damit die jungen Leute sie kaufen. (Unsichtbare Hände, Tietäväinen 2014: 16)

6. Talismane sind haram ${ }^{6}$. (Unsichtbare Hände, Tietäväinen 2104: 61)

Zum anderen werden für die Ausdrücke fremden Ursprungs zielsprachliche Entsprechungen oder Erklärungen im Panel, unterhalb des Panels oder in einer Fußnote gegeben. In Beispiel 7, das von Asterix bei den Briten herstammt, werden die deutschen Entsprechungen unterhalb des Panels gegeben $\left({ }^{*}\right.$ Lat. $=$ Lanze; ${ }^{* *}$ Lat. = Brustbein), in Beispiel 8, das auf Der Sohn des Asterix zurückgeht, dagegen im Text in Klammern sofort nach dem fremden Ausdruck. In Beispiel 9 ist für Schtetl* in der deutschen Ausgabe unterhalb des Panels die Erklärung „*Jiddische Dörfer in Osteuropa“ hinzugefügt worden.

7. Mein Garten ist kleiner als Rom, aber mein pilum* ist solider als euer sternum**. (Asterix bei den Briten, Goscinny/Uderzo 1985: 18)

8. Während die Erdarbeiter die Fossa (den Graben) ausheben und den Agger (den Wall) aufschaufeln... fällen die Holzhauer die Arbores (die Bäume)... die die Zimmerleute für das Vallum (die Palisade) brauchen! (Der Sohn des Asterix, Uderzo 2017: 24)

9. Sieht fast so aus wie die Schtetl* bej uns dahejm, findest du nicht, Rachel? (Lucky Luke, Achdé/Jul 2018: 24)

Hinsichtlich der Häufigkeit fremdsprachiger Ausdrücke stehen nach Wörtern und Wortgruppen fremden Ursprungs Phraseologismen an zweiter Stelle. Die fremdsprachigen Phraseologismen im untersuchten Korpus gehören haupt-

5 Die Djellaba „ist ein traditioneller bodenlanger und die Körperkonturen weitgehend verbergender Überwurf- und Kapuzenmantel mit langen Ärmeln [in den nordafrikanischen Territorien], insbesondere in Marokko". (Wikipedia, s. v. Djellaba)

6 Harām, ,ist ein arabisches Adjektiv, das im Islam alles dasjenige bezeichnet, was [...] unberührbar, unverletzlich, heilig, geheiligt oder aber verflucht, fluchbeladen bzw. verboten ist. In seiner Bedeutung ähnelt es im Deutschen am ehesten dem Begriff Tabu"e. (Wikipedia, s. v. Harām) 
sächlich zu den Anredeformeln (Beisp. 10, 11, 12, 22, Abb. 7) und den Routineformeln, die in verschiedene Hauptklassen und Subklassen eingeteilt werden können, wie z. B. in Grußformeln, d. h. Begegnungs- und Abschiedsformeln (Beisp. 10, 13, 14, 15, 16, 21, Abb. 7), emotive Formeln (Beisp. 17, 18, 19, 20), Dankesformeln (Beisp. 21), Entschuldigungsformeln (Beisp. 22), Wunsch- und Anlassformeln (Beisp. 23, 24) (zu Routineformeln ausführlicher z. B. Hyvärinen 2011: 23-34; Sosa Mayor 2006, zu emotiven Formeln Liimatainen 2011a, zu Wunsch- und Anlassformeln Liimatainen 2010).

10. Buenas tardes, señor Quijote.

(Unsichtbare Hände, Tietäväinen 2014: 32)

11. Danke, Sie sind sehr freundlich, Sidi.

(Unsichtbare Hände, Tietäväinen 2014: 25)

12. Ladies and Gentlemen, eine Ankündigung der Mississippi-DampfSchifffahrtsgesellschaft (Lucky Luke, Achdé/Jul 2018: 8)

13. Gut, ich gehe zu ihm. Salam!' (Tim und Struppi, Hergé 1998: 53)

14. Assalamu aleikum. - Wa aleikum assalam! - Dann kommt halt rein. (Unsichtbare Hände, Tietäväinen 2014: 111)

15. Salem aleikum! Ist es erlaubt einzutreten? (MOSAIK 2019, Heft 1: 3)

16. Merhaba ${ }^{8}$ (Hat man erst angefangen..., Beeler 2018: 37)

17. Mon Dieu! Wo bin ich denn? (Aristocats 1980: 9)

18. My God, eine Entführung! (Boule \& Bill, Duchateau et al. 1991: 24)

19. Ooooh! Ein Detektiv! Wonderful!

(Boule \& Bill, Duchateau et al.18991, 43)

20. $O j W e j^{* 9}$, hab ich den Namen von dem Cowboy vergessen, der uns abholen soll. (Lucky Luke, Achdé/Jul 2018: 10)

21. ... Gracias. Hasta luego. (Unsichtbare Hände, Tietäväinen 2014: 32)

7 Salām, ,ist ein üblicher Gruß im arabischen Sprachkontext [...]. Salām wird in der arabischen Formel [...] as-salāmu 'alaikum, Der Frieden auf Euch!’ als traditioneller Gruß unter Muslimen in der ganzen Welt gebraucht. Die übliche Antwort darauf ist [...] wa-'alaikumu s-salām, Und auf Euch der Frieden!"ee (Wikipedia, s. v. Saläm).

8 Eine türkische Grußformel ,Hallo! Guten Tag! (https://www.wortbedeutung. info, s. v. Merhaba).

$9 \quad$ Unterhalb des Panels: *Jiddischer Klageruf (Lucky Luke, Achdé/Jul 2018: 10). 
22. Huch! I am sorry, Madam! (Boule \& Bill, Duchateau et al. 1991: 42

23. MAZEL TOV!10 (Lucky Luke, Achdé/Jul 2018: 24)

24. BON VOYAGE (Maus II, Spiegelman 1998b: 125)

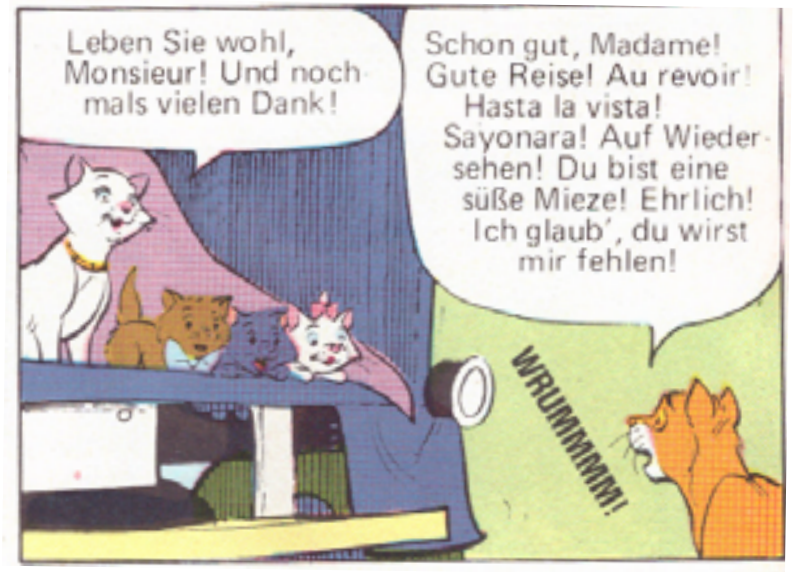

Abb 7: Aristocats

(1980: 16).

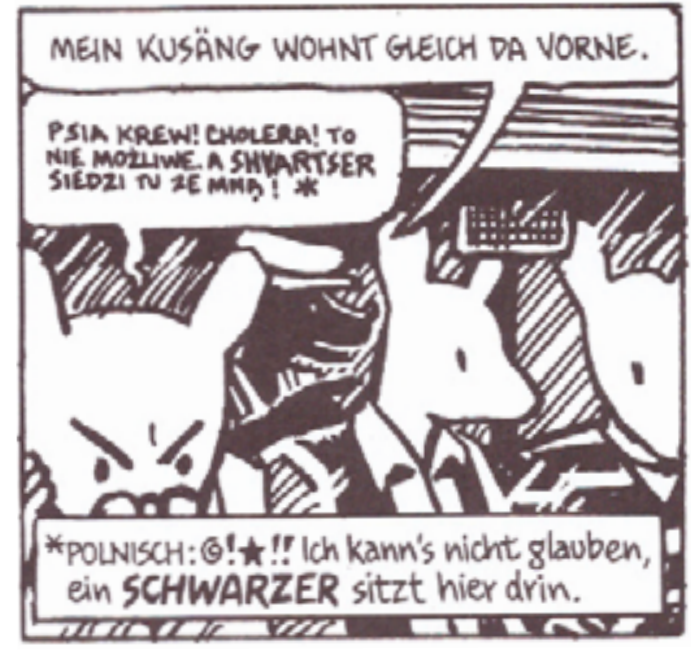

Abb. 8: Maus II (Spiegelman 1998b: 99).

In der Comic-Serie Asterix werden gelegentlich bekannte lateinische Zitate, d. h. geflügelte Worte verwendet. Ein geflügeltes Wort ist ein Ausdruck, der „auf eine bestimmte und allenfalls bestimmbare Quelle zurückgeht“ (Burger 2007: 49). Geflügelte Worte stammen aus der Literatur, aus historischen Dokumenten, aus Filmen, der Werbung oder anderen nicht-literarischen Bereichen der Sprache (ebd.). Es handelt sich um Ausdrücke, die im allgemeinen Sprachgebrauch eine generalisierte Bedeutung erhalten haben. Diese lateinischen Zitate werden in der französischen Ausgabe im Gegensatz zu Ausgaben in anderen Sprachen in Fußnoten nicht übersetzt (s. auch Mitrache 2004: 237-238 und Liimatainen 2020: 205-206). Diese Zitate sind in den ins Deutsche übersetzten Varianten mit einer Fußnote beibehalten, zusammen mit der deutschen Übersetzung ${ }^{11}$ : (Beisp. 25) *Der Würfel ist gefallen. (Asterix bei den Briten, Goscinny/Uderzo 1985: 14); (Beisp. 26): *Was zu beweisen war! (Der Sohn des Asterix, Uderzo 2017: 15).

10 Unterhalb des Panels: „Herzlichen Glückwunsch! auf Jiddisch“ (Lucky Luke, Achdé/ Jul 2018: 24).

11 Ähnlich auch in den finnischen Ausgaben (Liimatainen 2020: 205-206). 
25. Gut, gut! Alea jacta est*, wir fahren zurück nach Britannien. (Asterix bei den Briten, Goscinny/Uderzo 1985: 14)

26. Quod erat demonstrandum.* (Der Sohn des Asterix, Uderzo 2017: 15)

Im untersuchten Korpus tauchen auch formelhafte Kurztexte auf, zu denen abgeschlossene Texte gerechnet werden, die in bestimmten Situationen als ganze entweder unverändert oder in nahezu identischer Form immer wieder reproduziert werden (als Beispiele dienen PROHIBIDO ARROJAR BASURAY (Unsichtbare Hände, Tietäväinen 2014: 141) und !atencion! (Boule und Bill, Duchateau et al. 1991: 2) (zu formelhaften Kurztexten z. B. Liimatainen 2018). Fluchworte und adressatenspezifische Invektiven fremden Ursprungs kommen in mehreren der untersuchten Comics vor. Als Herkunfssprache tritt am häufigsten Englisch auf, gelegentlich auch andere Sprachen sowie künstliche Sprachen (Beispiele 27-30, Abb. 8).

27. Damned! Jemand hat die Brücke zerstört!

(Lucky Luke, Achdé/Jul 2018: 22)

28. Was ist denn? - Hijo de pu[ta $]^{12}$... Aaargh!

(Unsichtbare Hände, Tietäväinen 2014: 97)

29. Shit ... soll ich Zigaretten kaufen? (Comic Atlas Finnland 2014: 231)

30. Fuck you! Son of a bitch!! (Persepolis, Satrapi 2019: 321)

Gebete und Schlagertexte fremden Ursprungs spielen im gesammelten Material eine nicht unbedeutende Rolle. Sie lassen sich insbesondere in der Graphic Novel Unsichtbare Hände von Tietäväinen (2014) und im Album Lucky Luke: Das gelobte Land von Achdé/Jul (2018) nachweisen. Die Geschichte Unsichtbare Hände (Tietäväinen 2018) beginnt und schließt mit muslimischen Gebeten - einmal gesprochen auf der Überfahrt von Gibraltar nach Spanien auf nächtlicher See, ein andermal von der Spitze der Kolumbus-Säule in Barcelona aus. Diese Gebete sind in deutscher Übersetzung zu lesen. Auf der Überfahrt wird ein Gebet auch in madagassischer Sprache gesprochen (s. Abb. 9) und in

12 Hijo de pu[ta],Scheißkerl, Hurensohn, Dreckskerl, Schweinepriester, Schweinehund, de.pons.com/übersetzung/spanisch-deutsch/hijo+de+puta. 
der marokkanischen Hafenstadt Tanger hört man den islamischen Gebetsruf (Adhān), mit dem die Zeit zum Gebet bekannt gemacht wird (s. Abb. 10).
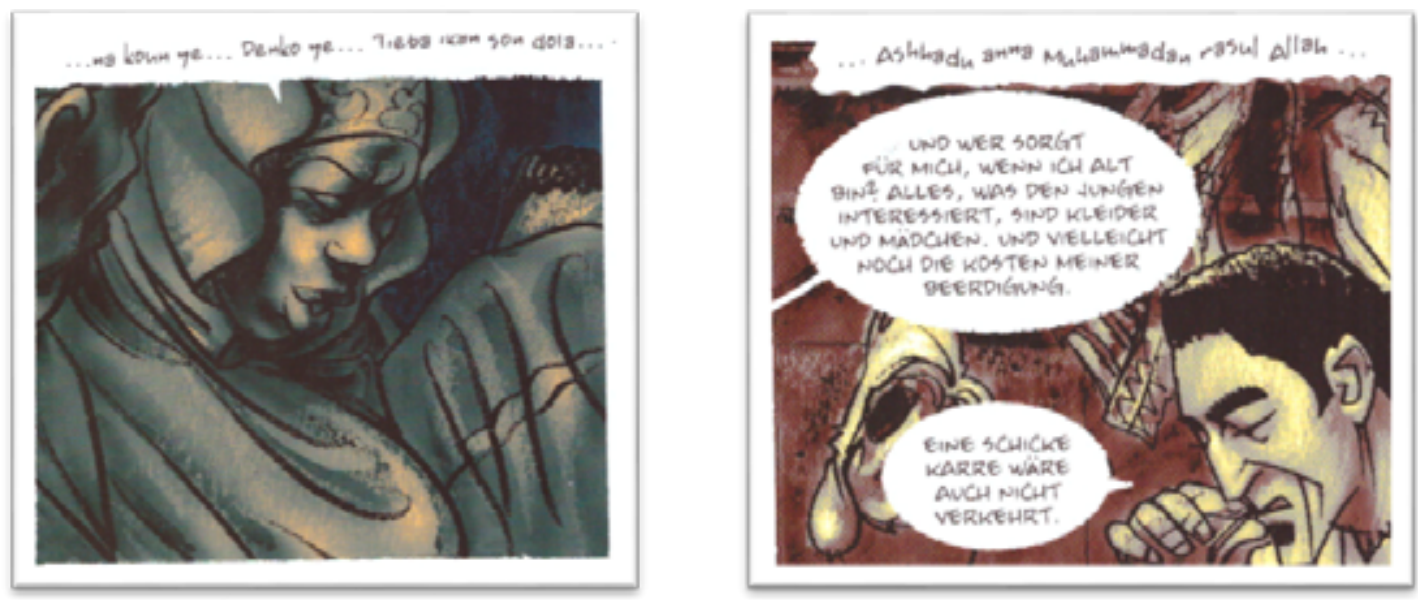

Abb. 9 und 10: Unsichtbare Hände (Tietäväinen 2014: 8, 24).

In Unsichtbare Hände von Tietäväinen (2014) kommen als Herkunftssprachen der Schlager das Spanische und das Finnische vor: Auf Seiten 32 bis 35 kann man den Liedtext des bekannten spanischen Schlagers La Tortura lesen, der im Autoradio gespielt wird. Auch die finnischen Schlager, gesungen von Jamppa Tuominen, erscheinen an mehreren Stellen (s. S. 142, 149, 151, 164 und 205) in ihrer Ursprungsform ohne entsprechende deutsche Übersetzung (Abb. 11). Die ausgangssprachliche Mehrsprachigkeit beizubehalten und eine zielsprachliche Version daneben zu drucken, wäre ein Kompromiss gewesen, der die künstlerische Qualität des Ausgangstextes bewahrt und gleichzeitig ein zielsprachliches Informationsangebot geliefert hätte.

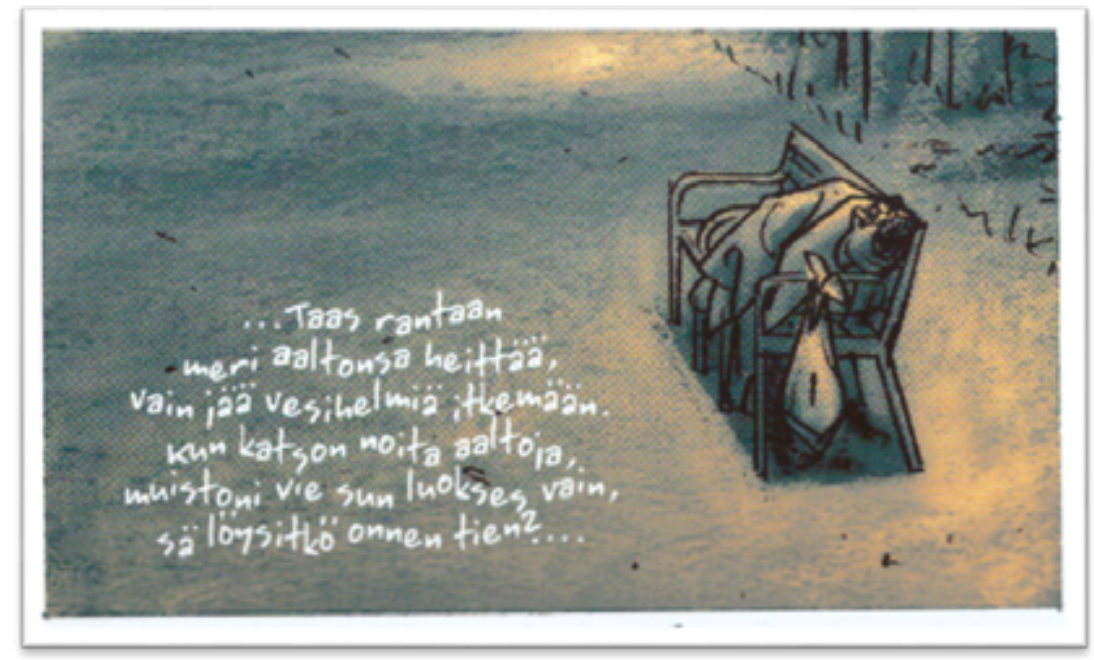

Abb. 11: Unsichtbare Hände (Tietäväinen 2014: 151). 
Der Band Das gelobte Land der Comic-Serie Lucky Luke (2018) beinhaltet zahlreiche Anspielungen auf das osteuropäische Judentum und die aschkenasische Kultur. In der deutschsprachigen Ausgabe sind als Herkunftssprachen neben dem Englischen auch die hebräische und die jiddische Sprache vorhanden. In zwei Sprechblasen befinden sich Texte (Gebete) mit hebräischem Schriftbild (s. Abb. 12) und auf Seite 36 singt Yankel Stern in mehreren Sprechblasen in jiddischer Sprache das Lied A Yiddishe Mame (Abb. 13) ohne Übersetzung in die Zielsprache in den Anmerkungen.
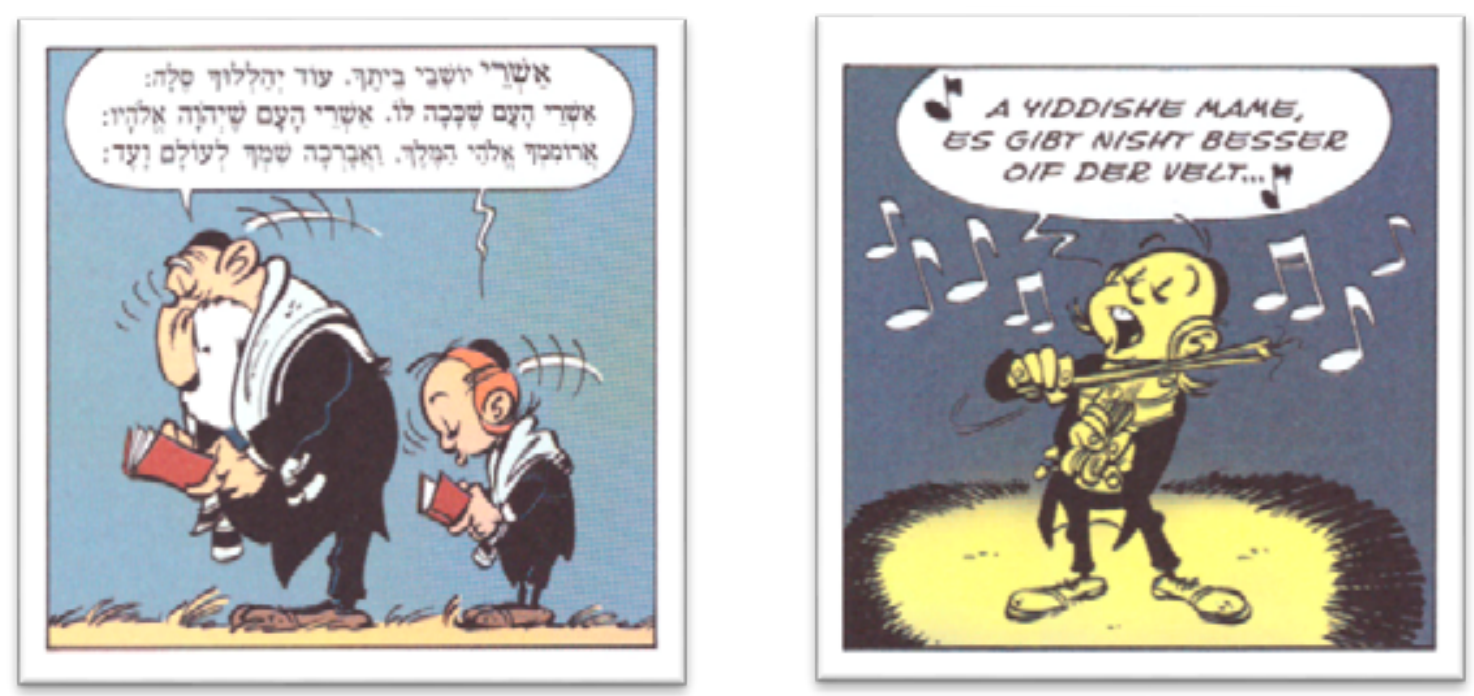

Abb. 12 und 13: Lucky Luke: Das gelobte Land (2018: 16, 36).

In den untersuchten Comics sind fremdsprachige Detailtexte üblich, die ausschlaggebend zur Vermittlung von Lokal- und Zeitkolorit der Geschichte beitragen oder den Geschichten Authentizität verleihen. Die besondere Atmosphäre des Schauplatzes spiegeln z. B. Ortsschilder wider, die in den Alben der Lucky Luke-Comics vorkommen. Die Schilder befinden sich im Allgemeinen am Eingang einer Stadt und teilen dem Besucher neben dem Ortsnamen häufig auch eine Warnung mit (Abb. 14). Die Asterix-Geschichten spielen laut der Einleitung um das Jahr 50 v. Chr. in Gallien, dem heutigen Frankreich, und damals wurden Orte und Staaten anders benannt als heutzutage. Diese Toponyme werden in deutschsprachigen Ausgaben in Fußnoten oder unterhalb des Panels übersetzt: Londinium=London, Mare Britannicum=Britannisches Meer, Portus Itius=Boulogne (Asterix bei den Briten 1985: 5, Abb. 15). Weitere Belege in Abb. 5 und 6. 


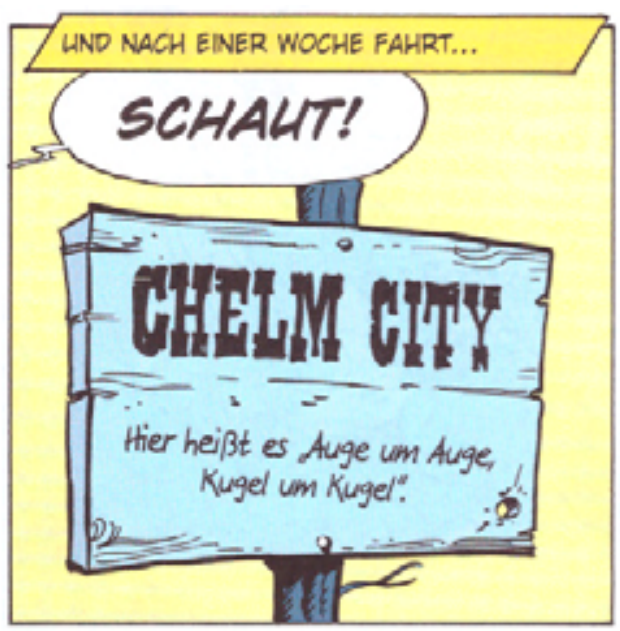

Abb. 14: Lucky Luke: Das gelobte Land (2018: 46).

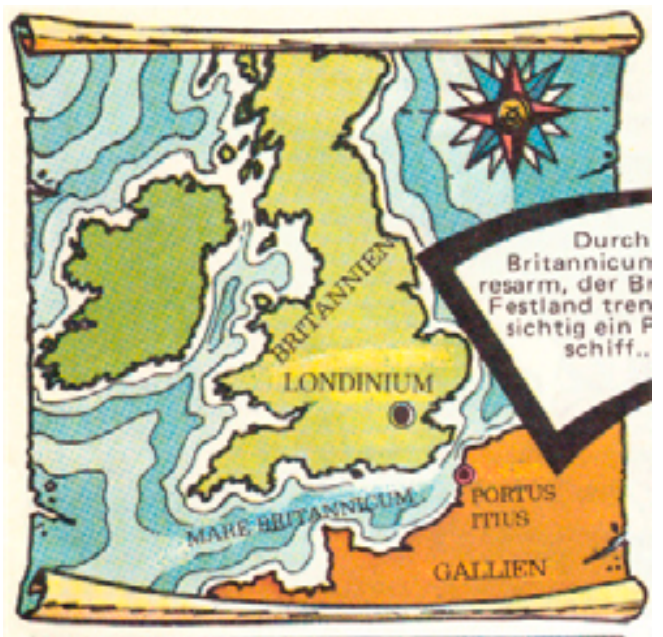

Abb. 15: Asterix bei den Briten (Goscinny/Uderzo 1985: 5).

In Persepolis von Sartrapi (2019) gibt es 24 Belege für persische Texte in Briefen, Zeitungen und Wandbildern, auf Schildern, Gebäudeaufschriften, Fahrzeugen, Grabsteinen, Mauern, an der Tafel Marjanes Klassenzimmer usw. (s. Abb. 16). Sie sind als Teile der Umgebung wichtige Bestandteile des Comicbildes und tragen entscheidend zur Vermittlung des Lokal- und Zeitkolorits im Iran bei.

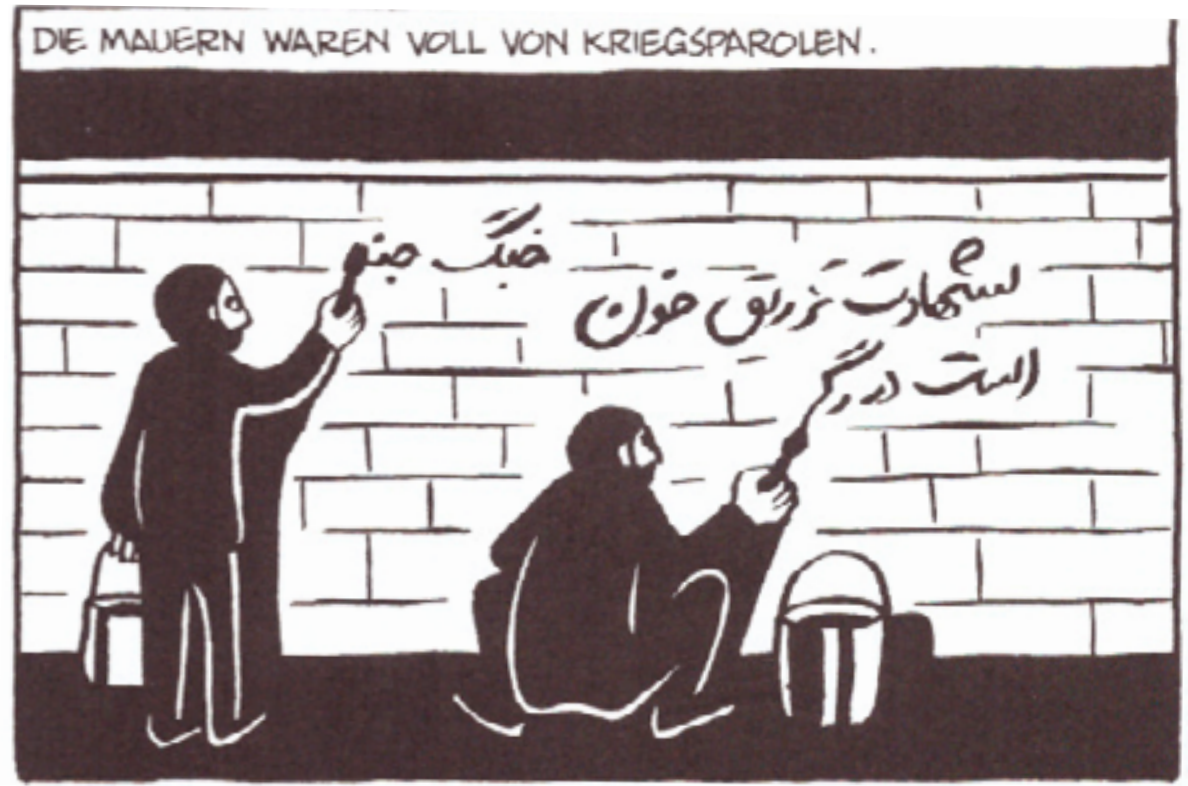

Abb. 16: Persepolis (Satrapi 2019: 117). 


\subsection{Kommunikative Funktionen der Mehrsprachigkeit}

Ein Comic kann nur dann als mehrsprachig bezeichnet werden, wenn die im Comic auftretenden Sprachen eine klare Funktion für die Geschichte haben und eine bestimmte Aufgabe erfüllen. Mehrsprachigkeit wird im untersuchten Korpus in erster Linie zur Vermittlung von Lokal- und Zeitkolorit bzw. zur räumlichen und zeitlichen Situierung der Handlung verwendet. Darüber hinaus dient Mehrsprachigkeit der Steigerung der Authentizität, z. B. durch die Färbung der Dialoge, der Widerspiegelung der besonderen Atmosphäre des Schauplatzes, der individualisierenden und typisierenden Figurencharakterisierung sowie der Aufwertung von den mit einer Region verbundenen Traditionen. Der Wortschatz kann eine auffallende Rolle bei der Personencharakterisierung spielen, indem der Autor Wörter, Anredeformeln, Fluchworte, Phraseologismen, Gebete, Lied- und Schlagertexte aus diversen anderen Sprachen aufnimmt.

In Unsichtbare Hände (Tietäväinen 2014) tauchen arabische Ausdrücke in Sprechblasen immer wieder auf, darüber hinaus spanische Entlehnungen sowohl in Sprechblasen als auch in Inserts. Sie verleihen der Geschichte Lokalkolorit, charakterisieren aber auch die afrikanischen Bootsflüchtlinge, die die Landessprache Spaniens nicht genügend beherrschen und auch deswegen ausgenutzt und schlecht behandelt werden. Mit Benennungen fremden Ursprungs kann Gewicht $u$. a. auch auf Abwertung einer ethnischen Gruppe gelegt werden. In Unsichtbare Hände wird die Hauptfigur Rashid von der spanischen Behörde Wetback genannt, was ursprünglich eine abwertende Bezeichnung für illegal in die USA eingewanderte Mexikaner ist (Beisp. 31).

31. Ha, von wegen! Du siehst doch schon an seinem gierigen Blick, dass das ein Wetback ist, der nur hinter dem schnellen Geld her ist. (Unsichtbare Hände, Tietäväinen 2014: 78)

In Das gelobte Land (Lucky Luke, Achdé/Jul 2018) sind die Mitglieder der aus Osteuropa kommenden jüdischen Familie Stern nicht nur durch ihre schwarze Kleidung und die Kopfbedeckungen zu erkennen, sondern sie werden auch durch die Beachtung des Sabbats und der jüdischen Essensrituale, das Verrichten der vorgeschriebenen Gebete und den Gebrauch der jiddischen Sprache von der Bevölkerung als fremdartig wahrgenommen. Es ist üblich, 
dass Comicfiguren sprachlich markiert werden, wenn sie einer bestimmten ethnischen Gruppe angehören, so beispielsweise auch die Cree-Indianer, die eine künstliche Sprache sprechen, in der sich im untersuchten Material Benennungen für Speisen mit Bezug zum Judentum verbergen (Lucky Luke, Achdé/Jul 2018: 43, 44, s. Beisp. 32 und Abb. 3).

\section{RASSRA, RASSRA OSBAN HARISSA! - MEKBOUBA? - MAKROUD DAFINA!13 (Lucky Luke, Achdé/Jul 2018: 43, 44)}

Ein typisches Kennzeichen von Jugendkulturen scheint zu sein, dass Texte und Titel der Songs überall in erster Linie in englischer Sprache sind. Dies beweisen auch die untersuchten Comics. Als Beispiele können der Comicstrip Dr. Bubi Livingston (Abb. 17) sowie die Graphic Novels Persepolis und Kinderland angeführt werden, die sich mit dem Leben von Jugendlichen in Deutschland sowie mit dem Leben von Kindern und Jugendlichen im Iran und in der DDR auseinandersetzen (Abb. 18 und 19).

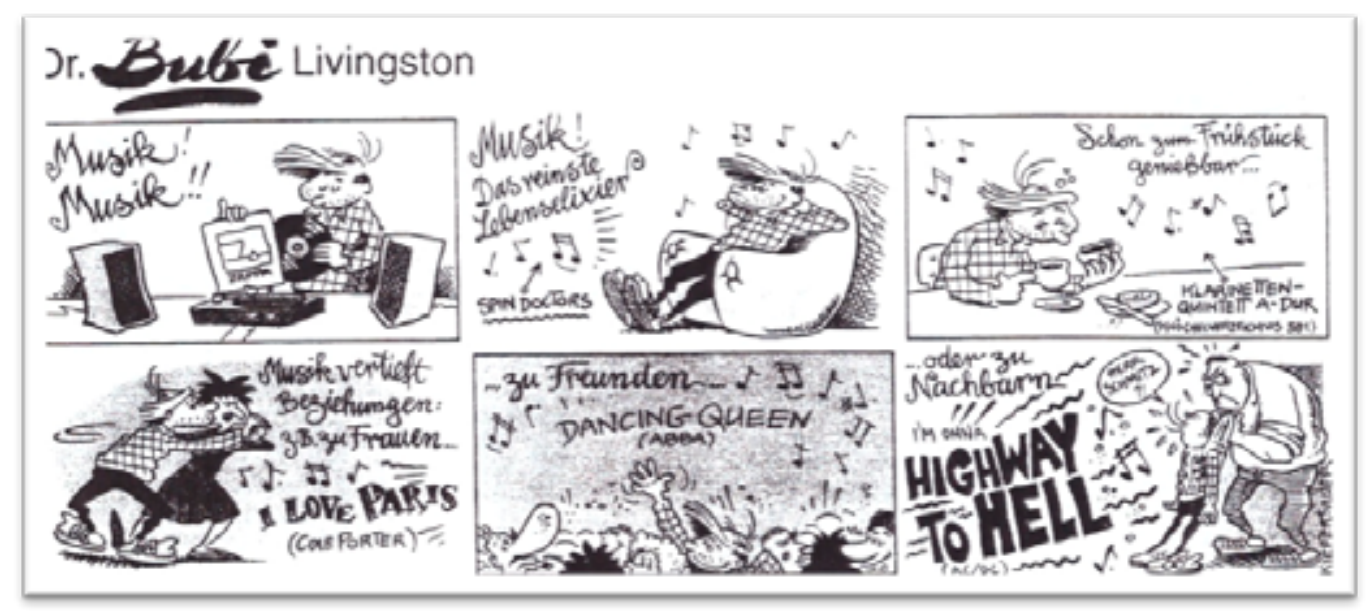

Abb. 17: Dr. Bubi Livingston (Kiefersauer 2000: Strip 96).

13 „Osban ist ein mit verschiedenen Zutaten wie Reis, Gewürzen, Lammfleisch, gehackter Leber und Herz gefüllter Pansen. Tunesische Harissa besteht aus grüner Paprika, Tomaten und Zwiebeln. Die Mekbouba ist ein auf Paprika und Tomaten basierendes Gemüsegericht tunesischer Juden. [...] Makroud ist ein süßes Gebäck mehrerer Länder Nordafrikas. [...] Dafina ist ein Schmorgericht aus Rindfleisch, Kartoffeln, Kichererbsen und weiteren Zutaten, das traditionell in der jüdischen Küche [...] zur zweiten Mahlzeit am Sabbat gehört." (Wikipedia, s. v. Das gelobte Land (Comic)) 


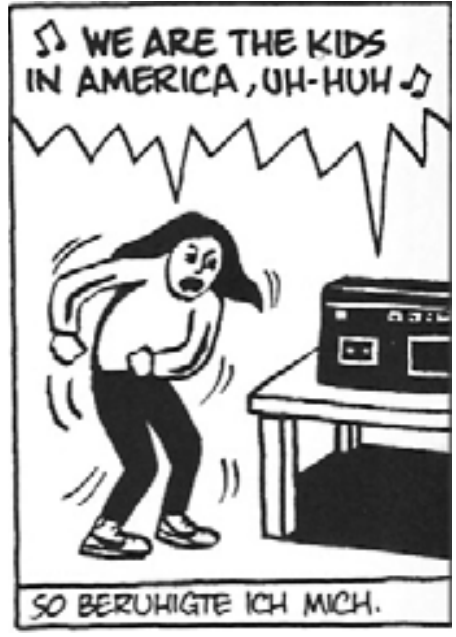

Abb. 18: Persepolis (Satrapi 2019: 136).

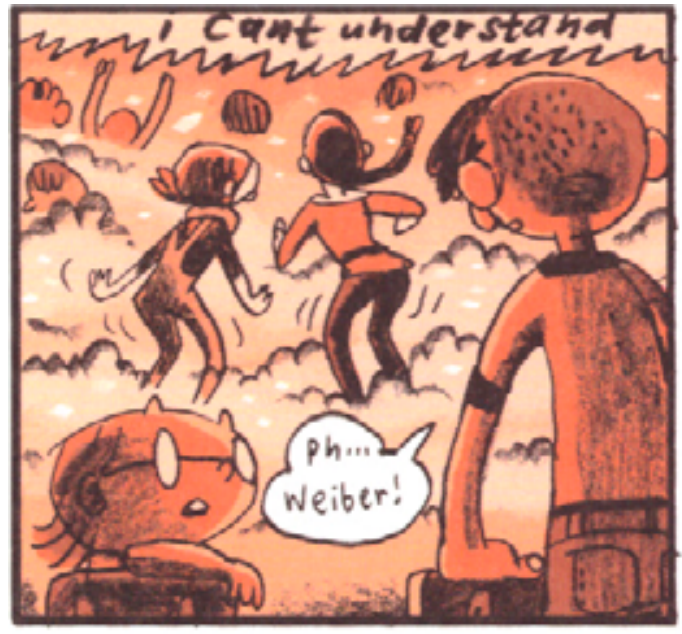

Abb. 19: Kinderland (Mawil 2017: 135).

Phraseologismen erfüllen in Comics gewisse Funktionen. Um das historische Milieu darzustellen und die antike, römische Atmosphäre nachzugestalten, werden in der Comic-Serie Asterix geflügelte Worte historischer Herkunft verwendet. Durch sie wird nicht selten auch das Komische der Situation ausgedrückt. (Vgl. auch Mitrache 2004: 237) Die Idiomatizität wird bewusst als Mittel zur Komik eingesetzt, insbesondere durch Modifikationen oder kontextuelle Verbindung zur wörtlichen Bedeutung. In der Zusammensetzung der lateinischen Wörter Argument (,etwas, was der Erhellung und Veranschaulichung dient') und baculinum (,Stock') wird in der scherzhaften Form wortwörtlich Ein schlagendes Argument! Dieser Ausdruck Argumentum baculinum ist im Album Der Sohn des Asterix einem Legionär in den Mund gelegt, als der und dessen Patrouille nach misslungener Volkszählung schon angeschlagen durch den Wald marschieren. Als dann noch der Sohn des Asterix seine Kräfte an Legionär Wurzelaus demonstriert, ergreift die ganze Gruppe panikartig die Flucht. Beim Versuch das Baby zu stillen, trinkt dieses versehentlich vom Zaubertrank, der ihm dann übermenschliche Kräfte verleiht. ${ }^{14}$ (Abb. 20) Die Situation ist bereits an sich komisch und das Komische wird anhand der Sprache noch vertieft.

14 Deutsches Asterix-Archiv (1998-2020). Lexikon, s.v Argumentum baculinum, https://www.comedix.de/lexikon/db/argument.php. 


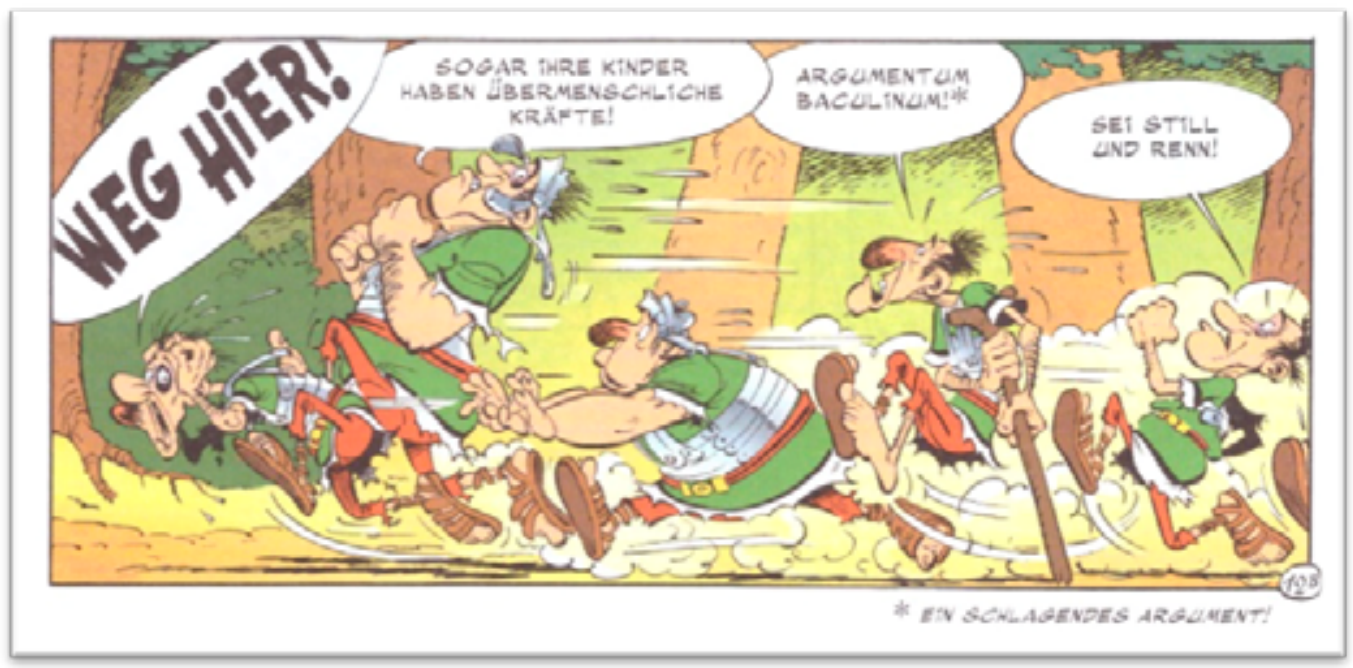

Abb. 20: Der Sohn des Asterix (Uderzo 2017: 16).

Im Album Die Schlümpfe (Peyo/Delporte 1983) werden lateinische Redewendungen kreativ verwendet, indem ein Teil des Phraseologismus durch das Einfügen des Wortes Schlumpf anstelle des richtigen Wortes ersetzt wird. Auf Seite 23 kommt in einer Sprechblase die modifizierte Redewendung Mea Schlumpfa! vor. Unterhalb des Panels steht eine weitere Modifikation „Oder Schlumfa culpa“. Als Basis der beiden Modifikationen wird die Redewendung Mea culpa verwendet, die ,Durch meine Schuld ' bedeutet. Auch auf Seite 33 wird die Modifikation einer Wortgruppe bewusst zur Komik eingesetzt: Schlumpf sana in corpore Schlumpfo. In diesem Beispiel wurden in der zugrunde liegenden lateinischen Redewendung Mens sana in corpore sano (,Ein gesunder Geist in einem gesunden Körper') die Wörter mens und sano durch Schlumpf und Schlumpfo ersetzt.

In mehreren der untersuchten Comics dienen Anredeformeln zur räumlichen Situierung der Handlung und zur Steigerung der Authentizität. Ungeachtet der Tatsache, dass Mangas äußerst kulturbeladen sind, finden sie auch außerhalb Japans als Übersetzungen großen Anklang. Die Anreden sind ein wesentlicher Teil der japanischen Höflichkeitssprache, und die Charaktere werden nach ihrem Namen z. B. mit Endungen -san,-kun, -chan und -sensei angeredet.

Bei der Anrede -sensei muss ein Name nicht unbedingt vorangestellt werden. Sensei wird z. B. für Lehrer (Mikami-sensei, eine Lehrerin an der Yomiyama-Mittelschule in Another 2nd Period 2017: 107), für Ärzte oder sonstige Autoritätspersonen genutzt. Die Anrede -san ist geschlechtsneutral, wird in 
erster Linie zwischen Erwachsenen verwendet, wenn man den Gesprächspartner kaum oder gar nicht kennt und entspricht den deutschen Anreden Frau und Herr. Die Anrede -kun kann sowohl mit dem Vor- (Koichi-kun) als auch mit dem Nachnamen (Sakakibara-kun, Another 2nd Period 2017: 37, 10) verwendet werden. Die Anrede -kun ist geläufig für Jungen und männliche Jugendliche. Lehrer hängen bei männlichen Schülern -kun an den Nachnamen an: „Kazami-kun und Akazawa-san werden als eure Vertreter an der Trauerfeier teilnehmen" (Another 2nd Period 2017: 110). Die Anrede -chan drückt eine enge Freundschaft oder ein hohes Maß an Zuneigung für die andere Person aus und findet Verwendung bei Babys, Kindern, jungen Mädchen, guten Freunden und Paaren: „Mei-chan, die Mitschülerin“ (Another 2nd Period 2017: 57). (Vgl. Balve 2019)

In der englischen Originalausgabe Maus. A Survivor's Tale (Spiegelman 2003) ist die deutsche Sprache eine der Fremdsprachen, die insbesondere die deutschen KZ-Wachleute, gelegentlich aber auch die Verfolgten und Gefangenen benutzen. Die deutsche Sprache spielt im Original eine auffallende Rolle, einerseits bei der Figurencharakterisierung, indem die Wachen die Befehle, die sie den Gefangenen hauptsächlich auf Englisch geben, häufig auch noch durch deutsche Ausdrücke verstärken (s. Beisp. 33-39). Befehle werden zum Teil in beiden Sprachen wiederholt, um dem Gesagten Nachdruck zu verleihen und die Aussage somit deutlicher zu machen. Andererseits werden im Original gewisse deutsche Benennungen für solche Begriffe verwendet, die typisch für die Zeit des Nationalsozialismus und der Judenverfolgung waren, z. B. Reichsmark, Dienst stadium [sic!], Judenrat, Arbeit macht frei, Reich, Blocksperre, Gestapo, Bettnachzieher (im Text die englische Entsprechung a bed-after-puller), Kraut (Spiegelman 2003: 79, 90, 114, 159, $192,219,222,227,271)$.

33. Halt, Jew! What are you carrying? (Spiegelman 2003: 87)

34. Er verblutete! His blod ran out! Carry him over to he truck with the others. (Spiegel-man 2003: 52)

35. JUDEN RAUS! (Spiegelman 2003: 115)

36. GET UNDRESSED! LEAVE YOUR VALUABLES! LINE UP! SCHNELL! (Spiegelman 2003: 185)

37. SCHNELL! SCHNELL! SCHNELL! (Spiegelman 2003: 186) 
38. EINS! ZWEI! DREI! (Spiegelman 2003: 217)

39. Halt or we'll shoot! (Spiegelman 2003: 266)

Der Protagonist Wladek Spiegelman wechselt die Sprache, um gewisse Figuren der laufenden Situation entweder aus- oder einzuschließen. So verwendet er Deutsch, wenn er sich vor seinen Verfolgern verstecken will. Beim Einsteigen in eine Straßenbahn, die nur für Deutsche und Polen bestimmt ist, grüßt er die anderen Reisenden mit Heil Hitler!, damit diese nicht merken, dass er Jude ist (Spiegelman 2003: 142). Ein anderes Mal muss er diesen Hitlergruß verwenden, wenn einige Kinder in Sosnowitz auf der Straße auf ihn zeigen und rufen, dass er Jude sei (Spiegelman 2003: 151). In Abb. 21 spricht Wladek mit seiner Frau und seinem Neffen und deren Frau Jiddisch, damit die polnischen Schmuggler sie nicht verstehen, wenn sie die Flucht aus Polen nach Ungarn planen.
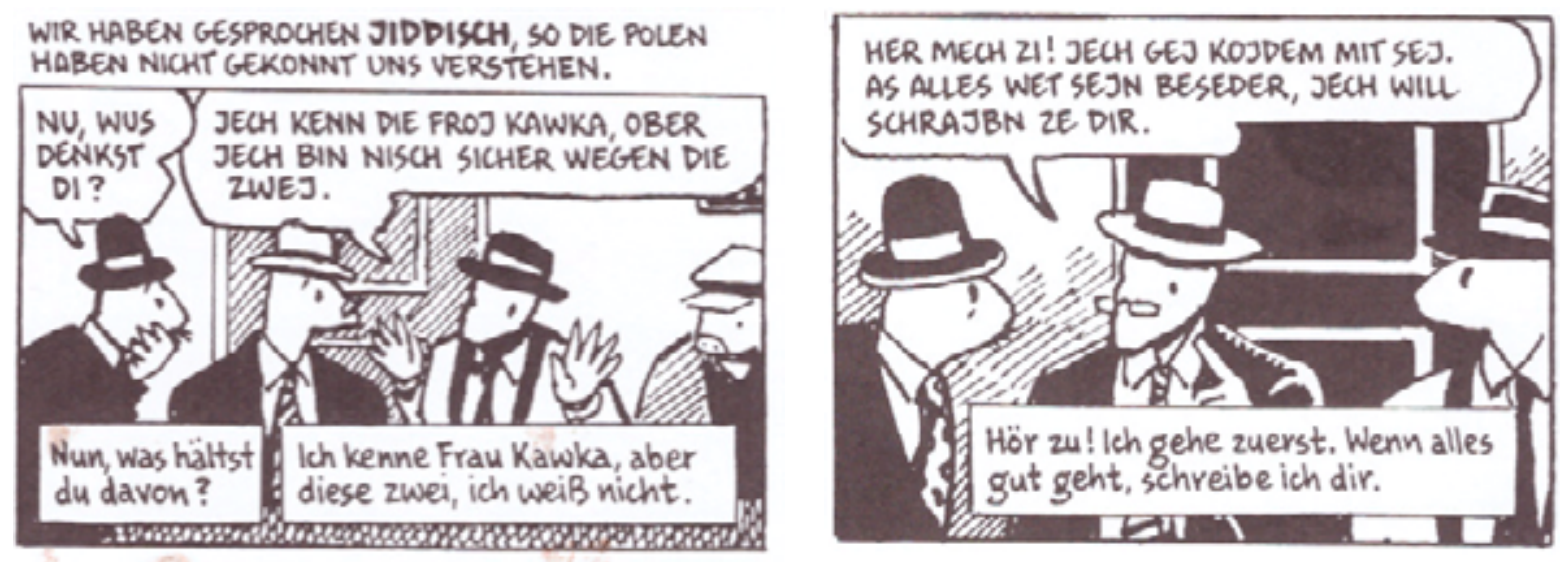

Abb 21: Maus I (Spiegelman 1998a: 150).

Wenn das Werk Maus, dessen Hauptsprache Englisch ist, in die Zielsprache Deutsch übersetzt wird, geht die Mehrstimmigkeit - eine Sprachebene des Werkes und ein Teil des Themas - zum großen Teil verloren. Mit Hilfe der deutschen Sprache wird in Maus die Geschichte in eine gewisse zeitliche, geschichtliche und sprachliche Umgebung integriert. Schließlich erhalten die Charaktere ihre Persönlichkeit nicht nur anhand der Illustration und der Ereignisse, sondern auch anhand ihres Sprachstils. Das Fehlen des CodeSwitching verflacht die Beschreibung, obwohl in der Übersetzung dieselben Inhalte erscheinen, die im Original vorhanden sind. Die deutsche Sprache im Original erzählt davon, dass die KZ-Aufseher Nazis sind, die Deutsch spre- 
chen. In der Übersetzung ins Deutsche sieht man diesen Unterschied nicht. In Bezug auf die Mehrsprachigkeit spielt Code-Switching also eine wichtige Rolle. Somit bleiben diejenigen Effekte, die beim Original mit der Mehrsprachigkeit erzielt wurden, in der einsprachigen Übersetzung verdeckt.

\section{Schlussbemerkungen}

Trotz des begrenzten, aber jedoch recht vielseitigen und repräsentativen Umfangs des untersuchten Materials (insgesamt 2614 Seiten), das sowohl original deutschsprachige Comics als auch Übersetzungen aus den frankobegischen und amerikanischen Sprachräume sowie aus dem Finnischen und Japanischen umfasst, zeigen die Ergebnisse des vorliegenden Artikels, dass die Sprache des Comics dazu herangezogen werden kann, Sprachwandelprozesse zu beleuchten und zu klären. Ziel der Untersuchung war es nicht, universelle Kriterien der Mehrsprachigkeit festzulegen, stattdessen jedoch aufzuzeigen, was alles zur Mehrsprachigkeit in Comics gehört und welche Funktionen sie in Comics haben kann.

Zusammenfassend kann zuerst festgestellt werden, dass Mehrsprachigkeit in neueren, insbesondere in übersetzten Comics häufiger als in Comics älteren Datums wie auch in original deutschsprachigen Comics auftritt. Dies trifft aber nicht konsequent auf alle Comics zu, denn es scheint auch von der Ausgangssprache und Ausgangskultur abzuhängen, wie hoch sich die Mehrsprachigkeit nachweisen lässt. Dies ist auch von Liimatainen (2020) nachgewiesen worden, die die Mehrsprachigkeit in deutschen und finnischen Comics kontrastiert hat. In Comics, die in erster Linie für Kinder und Jugendliche gedacht sind, tritt Mehrsprachigkeit scheinbar weniger auf als in Comics, die sich hauptsächlich auf die Zielgruppe von Erwachsenen konzentrieren. Von einer übermäßigen Verwendung von Ausdrücken fremden Ursprungs kann den empirischen Daten nach jedoch keine Rede sein. Darüber hinaus wird vom ZS-Leser keine allzu große sprachliche Kompetenz erwartet, denn am allermeisten sind die Ausdrücke fremden Ursprungs einzelne Wörter, Routineformeln oder Song- und Schlagertexte, die dem Leser das Verständnis des Inhalts der Geschichte nicht deutlich erschweren.

Da Anglizismen heute beinahe alle Lebensbereiche durchdringen, war es zu erwarten, dass das Englische als Gebersprache dominiert. Wie in der 
Analyse gezeigt wird, macht sich neben den Schulfremdsprachen der Einfluss von Migrantensprachen als Herkunftssprachen in jüngster Zeit auch in Comics verstärkt bemerkbar.

Im Bereich der Entlehnungen beschränkt sich der Einfluss von Fremdsprachen nicht nur auf die Übernahme von einzelnen Wörtern. Immer häufiger kommen Phraseologismen, Anredeformeln, Fluchwörter, Invektiven, Gebete und Schlager fremden Ursprungs vor. Die Entlehnungen verleihen den Geschichten Authentizität sowie Lokal- und Zeitkolorit. Sie sind wichtig auch für die Charakterisierung von historischen Handlungsorten, von Personen des Werkes sowie ihrer Beziehungen untereinander. Gegenspieler und Randfiguren werden sprachlich markiert, wenn sie einer bestimmten ethnischen Gruppe angehören. Mehrsprachigkeit kann häufig auch als Quelle für Komik verwendet werden. Eine entscheidende Rolle spielt sie für die Gesamtbedeutung des Werkes.

Die Vielfalt von Kulturen und Kulturspezifika, die Mehrsprachigkeit, Sprachspiele sowie das Zusammenspiel zwischen Sprache und Bild stellen bei allen Arten von Comics die größten Übersetzungsprobleme dar. Aus translatorischer Sicht ist es daher wichtig, dass bei der Übersetzung von Comics auch diese Vielfalt spürbar gemacht und die Mehrsprachigkeit des Ausgangstextes in Betracht gezogen wird, da sonst ein Teil der Gesamtinformation und die Mehrstimmigkeit verloren gehen. Im untersuchten Korpus wird die Mehrsprachigkeit hauptsächlich unübersetzt beibehalten. In vielen Fällen wird eine zielsprachliche Übersetzung im Text in Klammern, unterhalb des Panels oder in einer Fußnnote angeführt, insbesondere wenn es sich um eine für den Handlungsverlauf der Geschichte wichtige Information handelt. $\mathbf{v}$

\author{
ANNIKKI LIIMATAINEN \\ UNIVERSTITÄT TAMPERE
}




\section{Bibliographie}

\section{Primärliteratur}

Aristocats 1980. Die schönsten

Disney-Geschichten 10. Stuttgart:

Ehapa Verlag.

AYATSUJI, Yukito/Kiyohara, Hiro 2017.

Another 2nd Period. 8. Aufl. Orig. „Another" Vol. 02, 2011. Aus dem Japanischen von Ai Aoki. Berlin: Egmont Manga.

BARKS, Carl 1994. Donald Duck 2. Barks Library. Aus dem Amerikanischen von Dr. Erika Fuchs. Stuttgart: Ehapa Verlag. BEELER, Pirmin 2018. Hat man erst angefangen zu reden, kann alles Mögliche dabei herauskommen. Zürich: Edition Moderne. DONNER, K[arl]-H[einz]/Blasco Jesus [1986]. Birbad 1: Das Germanendorf. Augsburg: BIRBAD-Comic Verlags $\mathrm{GmbH}$.

DUCHATEAU, André-Paul et al. 1991. Ein aussergewöhnliches Abenteuer von Boule und Bill: Bill ist fort! Aus dem Französischen von Petra Weilbächer. Stuttgart: Ehapa Comic Collection. GOSCINNY, [René]/Uderzo, [Albert] 1985. Asterix bei den Briten. Orig. Astérix chez les bretons 1966. Übersetzung Gudrun Penndorf. Stuttgart: Delta. (Erste Veröffentlichung in deutscher Sprache 1971).

HANNA [William]/Barbera [Joseph] 1974.

Familie Feuerstein 3: Jagd auf den Steinhüpfer. Hamburg: Neuer Tessloff Verlag.

HERGÉ [Remi, Georges Prosper] 1998. Tim und Struppi: Die Krabbe mit den goldenen Scheren. Orig. Le crabe aux pinces d'or 1941. Aus dem Französischen von Ilse Strasmann.
Hamburg: Carlsen. (Erste Veröffentlichung in deutscher Sprache 1943).

HINRICHER, Jens/Kess, Martin/Breitschuh, Eckart 1994. Das Geheimnis der Lindenstraße. Erster Teil: Dresslers letzte Fahrt. 5. Aufl. Hamburg: Carlsen. HOMMER, Sascha/Hakkola, Kalle (Hrsg.) 2014. COMIC ATLAS FINNLAND.

Aus dem Finnischen von Elina

Kritzokat. Berlin: Reprodukt. JANSSON, Tove 2015. Mumins: Die gesammelten Comic-Strips von Tove Jansson 3. Aus dem Englischen von Annette von der Weppen, Michael Gronewald und Matthias Wieland. Berlin: Reprodukt.

KIEFERSAUER, Johann 2000. Dr. Bubi Livingston. Du! Du! Du! ICH! ICH! ICH! Berlin: JOCHEN enterprices. KÖNIG, Ralf 1987. Der bewegte Mann. Reinbeck bei Hamburg: Rowohlt. Lucky Luke: Das gelobte Land 2018. Zeichnungen: Achdé, Text: Jul nach Morris. 3. Aufl. Orig. Lucky Luke: La Terre Promise 2016. Aus dem Französischen von Klaus Jöken. Berlin: Egmont MAWIL 2017 = Witzel, Markus Mawil 2017. Kinderland. 5. Aufl. Berlin: Reprodukt. MOSAIK-Sammelband 19. 2019.

Wüstenräuber. 3. Aufl. Hrsg. von Klaus D. Schleiter. Die Erstveröffentlichung der in diesem Sammelband abgedruckten Abrafaxe-Abenteuer erschien 1982 in den MOSAIK-Heften 1 bis. 4. Berlin: Steinchen für Steinchen Verlag. PEYO [Culliford, Pierre]/Delporte, Y[van] 1983. Die Schlümpfe 6: Rotschlümpfchen und Schlumpfkäppchen. Orig. 
Schtroumpfvert et vert Schtroumpf

1978. Aus dem Französischen von

Sigrid von Fischern. Reinbeck bei

Hamburg: Semic.

SATRAPI, Marjane 2019. Persepolis.

Gesamtausgabe. 6. Aufl. Orig.

Persepolis, 2000-2003. Übersetzung:

Stephan Pörtner. Zürich: Edition

Moderne.

SPIEGELMAN, Art 1998a. Maus I. Die

Geschichte eines Überlebenden. Mein

Vater kotzt Geschichte aus. Orig. Maus.

A Survivor's Tale 1986. Deutsch von

Christine Brinck und Josef Joffe.

Reinbek bei Hamburg: Rowohlt.

SPIEGELMAN, Art 1998b. Maus II. Die

Geschichte eines Überlebenden. Und

hier begann mein Unglück. Orig. Maus.

A Survivor's Tale II; And Here My

Troubles Began 1991. Deutsch von

Christine Brinck und Josef Joffe.

Reinbek bei Hamburg: Rowohlt.

TIETÄVÄINEN, Ville 2014. Unsichtbare

Hände. Orig. Näkymättömät kädet

2011. Übersetzung von Alexandra

Stang. Berlin: avant-verlag.

UDERZO, Albert 2017. Der Sohn des

Asterix. Orig. Le Fils d'Astérix 1983.

Aus dem Französischen von Gudrun

Penndorf. Berlin: Egmont Ehapa.

(Erste Veröffentlichung in deutscher

Sprache 1983).

Woody Woodpecker [1977]. Neue lustige

COMIC-Abenteuer von Woody und

seinen Freunden. Comic-Album Nr. 1.

Frankfurt a. M.: Condor Print \& Verlag

$\mathrm{GmbH} \& \mathrm{Co}$. KG.

\section{Sekundärliteratur}

BACCOLINI, Raffaella/Zanettin, Federico

2014. The Language of Trauma. Art

Spiegelman's Maus and its

Translations. Comics in Translation, Hrsg. Federico Zanettin. London/New

York: Routledge. 99-132.
BALVE, Maren 2019. Sama, san, kun, chan: Die japanische Anrede einfach erklärt. GO! GO! NIHON. https://gogonihon.com/ de/blog/sama-san-kun-chan-diejapanische-anrede-erklaert/.

BASTIAN, Sabine 2013. Fluchen und Schimpfen im Comic - Von Kapitän Haddock zu den Banlieu-Zards. Alles hängt mit allem zusammen. Translatologische Interdependenzen. Festschrift für Peter A. Schmitt, Hrsg. Anne-Kathrin Ende/Susann Herold/ Annette Weilandt. Berlin: Frank \& Timme. 453-467.

BURGER, Harald 2007. Phraseologie. Eine Einführung am Beispiel des Deutschen. 3., neu bearb. Aufl.

Berlin: Schmidt Verlag. BUSCH, Brigitta 2013. Mehrsprachigkeit. Wien: Facultas.

DIAZ CINTAS, Jorge 2011. Dealing with Multilingual Films in Audiovisual Translation. TranslationSprachvariation-Mehrsprachigkeit. Festschrift für Lew Zybatow zum 60. Geburtstag, Hrsg. Wolfgang Pöckl/ Ingeborg Ohnheiser/Peter Sandrini. Frankfurt a. M. etc.: Lang. 215-233. ENELL-NILSSON, Mona/Hjort, Minna 2013.

"Fuck sådant." Snabba cash -romaanin monikielisistä voima- ja haukkumasanoista sekä niiden suomen-, saksanja englanninkielisistä käännöksistä. MikaEL. Kääntämisen ja tulkkauksen tutkimuksen symposiumin verkkojulkaisu 7. Electronic proceedings of the KäTu symposium on translation and interpreting studies 7, Hrsg. Marja Kivilehto/Minna Ruokonen/Leena Salmi. Helsinki: SKTL. 95-110.

FIEDLER, Sabine 2016a. Der Comic als Reflexion von Zeitgeschichte und Sprachzustand. Sprache im ComicIl linguaggio dei fumetti - La lengua de los cómics, Hrsg. Hartmut E. H. Lenk/ 
Elina Suomela-Härmä. Helsinki: Société Néophilologique. 135-158. FIEDLER, Sabine 2016b. Mehr als KRACH, PENG, ARRRRRGH - die Sprache des Comics. Sprache im Comic Il linguaggio dei fumetti - La lengua de los cómics, Hrsg. Hartmut E. H. Lenk/ Elina Suomela-Härmä. Helsinki: Société Néophilologique. 29-48. GoscinNY, René/Uderzo, Albert 2004. Veni, vidi, vici: das grosse AsterixLatinum. Lateinüberlieferungen in Wort und Bild. Ich kam, sah und siegte. Übers. der aus den AsterixAbenteuern verwendeten Bildzitate aus dem Franz. von Gudrun Penndorf. Köln: Egmont Ehapa Verlag. GRUTMAN, Rainier 2006. Refraction and Recognition: Literary Multilingualism in Translation. Target 18 (1): 17-47. https://doi.org/10.1075/target.18.1.03gru GRUTMAN, Rainier 2009. Multilingualism. Routledge Encyclopedia of Translation Studies, Hrsg. Mona Baker/Gabriela Saldanha. $2^{\text {nd }}$ Ed. London/New York: Routledge. 182-185.

HYVÄRINEN, Irma 2011. Zur Abgrenzung und Typologie pragmatischer Phraseologismen - Forschungsüberblick und offene Fragen. Beiträge zur pragmatischen Phraseologie, Hrsg. Irma Hyvärinen/Annikki Liimatainen. Frankfurt a. M. etc.: Lang. 9-43. JÜNGST, Heike 2014. Translating Manga. Comics in Translation, Hrsg. Federico Zanettin. London/New York:

Routledge. 50-78.

KAINDL, Klaus 2004.

Übersetzungswissenschaft im interdisziplinären Dialog. Am Beispiel der Comicübersetzung. Tübingen: Stauffenburg. KAINDL, Klaus 2010. Comics in translation. Handbook of Translation Studies 1, Hrsg. Yves Gambier/Luc van Doorslaer. Amsterdam/Philadelphia:
John Benjamins. 36-40. https://doi.org/10.1075/hts.1.comi1 KEROMNES, Yvon 2019. Idiomatisch und kreativ? Comics in der Übersetzung. Approches théoriques et empiriques en phraséologie / Therie und Empirie in der Phraseologie. https://hal.archivesouvertes.fr/hal-03030532.

LIIMATAINEN, Annikki 2010. Alles Liebe und Gute! Wunsch- und Anlassformeln im deutsch-finnischen Kontrast. Infinite Kontrastive Hypothesen. Beiträge des Festsymposiums zum 60. Geburtstag von Irma Hyvärinen, Hrsg. Leena Kolehmainen/Hartmut E. H. Lenk/Annikki Liimatainen. Frankfurt a. M etc.: Lang. 165-183.

LIIMATAINEN, Annikki 2011a. Ach du Donnerchen! - Voi herran pieksut! Zur Wiedergabe der emotiven Formeln in deutschen und finnischen Übersetzungen. Beiträge zur pragmatischen Phraseologie, Hrsg. Irma Hyvärinen/Annikki Liimatainen. Frankfurt a. M. etc.: Lang. 113-145.

LIIMATAINEN, Annikki 2011b. Das Problem der Übersetzbarkeit von Fluchformeln. Multi-Lingual Phraseography: Second Language Learning and Translation Applications, Hrsg. Antonio Pamies/Lucía Luque Nadal/José Manuel Pazos Bretaña. Baltmannsweiler: Schneider Verlag Hohengehren. 265-272.

LIIMATAINEN, Annikki 2013. Zeitbindung bei Erst- und Neuübersetzung von Phraseologismen. Phraseologie im interlingualen und interkulturellen Kontakt - Phraseology in Interlingual and Intercultural Contact, Hrsg. Melanija Fabčič/Sabine Fiedler/Joanna Szerszunowicz. Maribor: Univerza v Mariboru. 147-160.

LIIMATAINEN, Annikki 2015. Thomas Mannin Buddenbrookit uudelleenkääntämishypoteesin näkökulmasta. 
Kontextwechsel-Zur gegenseitigen Vermittlung finnischer und deutscher Literatur durch Übersetzung/ Kontekstinvaihto - käännökset suomalaisen ja saksalaisen kirjallisuuden välittäjinä, Hrsg. Marja Järventausta/Liisa Laukkanen/ Christoph Parry. München: iudicium. 84-105.

LIIMATAINEN, Annikki 2018. Formelhafte Kurztexte: Bahnhofs- und Zugansagen. Phraséologie et discours, Hrsg. Olivier Soutet/Inès Sfar/Salah Mejri. Paris: Honoré champion. 179-191.

LIIMATAINEN, Annikki 2020. Mehrsprachigkeit und Multikulturalität in Comics als Ausdruck des gesellschaftlichen Wandels. Themenheft „Mehrsprachigkeit Transkulturalität - Identitäten ", Gastherausgeber Ewald Reuter. German as a foreign language 3: 185-217. http://www.gfl-journal.de/. MELONI, Ilaria 2016. Die Micky-MausÜbersetzungen von Erica Fuchs: Ein Beitrag zur deutschen Sprache? Sprache im Comic - Il linguaggio dei fumetti-La lengua de los cómics, Hrsg. Hartmut E. H. Lenk/ Elina Suomela-Härmä. Helsinki: Société Néophilologique. 159-186.

MEYLAERTS, Reine 2010. Multilingualism and translation. Handbook of Translation Studies 1, Hrsg. Yves Gambier/Luc van Doorslaer. Amsterdam/Philadelphia: John Benjamins. 227-230. https://doi.org/10.1075/hts.1.mul1 MITRACHE, Liliana 2004. Die Problematik der Übersetzung von Phraseologismen in Comics. Eine Untersuchung anhand der Übersetzungen von Asterix ins Deutsche und Schwedische. Phraseologismen als Gegenstand sprach-und kulturwissenschaftlicher Forschung. Akten der Europäischen
Gesellschaft für Phraseologie (EUROPHRAS) und des Westfälischen Arbeitskreises "Phraseologie/ Parömiologie« (Loccum 2002), Hrsg. Csaba Földes/Jan Wirrer. Baltmannsweiler: Schneider Verlag Hohengehren. 227-239.

NEULAND, Eva 2007. Mehrsprachig kontrastiv - interkulturell: Zur Heterogenität und Typizität von Jugendsprachen. Jugendsprachen: mehrsprachig - kontrastivinterkulturell, Hrsg. Eva Neuland. Frankfurt a. M. etc.: Lang. 11-29. NEULAND, Eva/Schubert, Daniel/Steffin, Hanne 2007. Ciao, salut, hadi und bye. Internationalismen im Sprachgebrauch Jugendlicher? Jugendsprachen: mehrsprachig - kontrastiv interkulturell, Hrsg. Eva Neuland. Frankfurt a. M. etc.: Lang. 117-134. NURMI, Arja 2013. Monikielisen tekstin kääntämisen strategiat: tapaustutkimus. MikaEL. Kääntämisen ja tulkkauksen tutkimuksen symposiumin verkkojulkaisu 7. Electronic proceedings of the KäTu symposium on translation and interpreting studies 7 , Hrsg. Marja Kivilehto/Minna Ruokonen/Leena Salmi. Helsinki: SKTL. 111-123.

NURMINEN, Laura 2013. Code-switching and non-standard language in the Finnish translations of African and Caribbean novels from the 1950s to the 2000s. MikaEL. Kääntämisen ja tulkkauksen tutkimuksen symposiumin verkkojulkaisu 7. Electronic proceedings of the KäTu symposium on translation and interpreting studies 7, Hrsg. Marja Kivilehto/Minna Ruokonen/Leena Salmi. Helsinki: SKTL. 124-134.

ossmanN, Andrea 2004. Phänomen Manga. Die Entstehungsgeschichte japanischer Comics und ihre 

und ins Deutsche übersetzten Comics im Wandel der Zeit • https://doi.org/10.51814/nm.103233

Bedeutung für deutsche Verlage und Bibliotheken. Mit einer annotierten Titelliste. Diplomarbeit im Studiengang Bibliotheks- und Medienmanagement an der Fachhochschule Stuttgart - Hochschule der Medien. https://hdms.bsz-bw.de/frontdoor/ deliver/index/docId/385/file/manga.pdf. PETERSEN, Robert S. 2021. Die Geschichte des Comics - Das Elitäre in den Künsten. Goethe-Institut USA. www.goethe.de/ins/us/de/kul/art/ pop/21447842.html.

RIEHL, Claudia Maria 2013. Mehrsprachigkeit und Sprachkontakt. Sprachwissenschaft: GrammatikInteraktion - Kognition, Hrsg. Peter Auer. Stuttgart/Weimar: Metzler. 377-404. https://doi.org/ 10.1007/978-3-476-00581-6_11 RÖMPÖTTI, Harri 2017. Sarjakuva sensuurin kourissa. Helsingin Sanomat 21.11.2017: B5.

SAARI, Mirja 2007. Code-switching im Kontext von Zweisprachigkeit am Beispiel von Jugendlichen in Helsinki. Jugendsprachen: mehrsprachig kontrastiv - interkulturell, Hrsg. Eva Neuland. Frankfurt a. M. etc.: Lang. Eva Neuland (Hrsg.). 179-194. SCHMITT, Peter A. 1997. Comics und Cartoons: (k)ein Gegenstand der Übersetzungswissenschaft? Transfer: Übersetzen-Dolmetschen-Interkulturalität, Hrsg. Horst W. Drescher. Frankfurt a.M. etc.: Lang. 619-662. SCHMITT, Peter A. 2003. Graphische Literatur, Comics. Handbuch Translation, Hrsg. Mary Snell-Hornby et al. 2., verb. Aufl. Tübingen: Stauffenburg. 266-269.

SCHWARZ, Alexander 2004. Sprachwissenschaftliche Aspekte der Übersetzung von Comics. Übersetzung: Ein internationales Handbuch zur Übersetzungsforschung, Hrsg. Harald
Kittel et al. Berlin/New York: de Gruyter. 676-683. https://doi.org/ 10.1515/9783110137088.1.7.676

SOSA MAYOR, Igor 2006. Routineformeln im Spanischen und im Deutschen. Eine pragmalinguistische kontrastive Analyse. Wien: Praesens.

SPIEGELMAN, Art 2003. Maus.

A Survivor's Tale. The Complete Maus: I My Father Bleeds History, II, And Here My Troubles Began. London: Penguin Books.

TABELING, Petra 2007. Das schreckliche Mädchen aus Teheran. Rezension in Neue Zürcher Zeitung 26.3.2007.

https://www.nzz.ch/ articleEX9VY-1.133755.

THUREN, Julia 2018. Uljas uusi sarjakuva. Helsingin Sanomat NS NYT 25.-31.5.2018, 2-4.

TOLVANEN, Juhani 2015. Der Comic vervollständigt das Universum. Orig. Sarjakuva täydentää universumin 2010. Aus dem Finnischen von Elina Kritzokat. Mumins. Die gesammelten Comic-Strips von Tove Jansson 3. Berlin: Reprodukt. 107.

TÖRNE, Lars von 2016. „Akira““, „Dragon Ball“ und „Sailor Moon“. Wie der Manga nach Deutschland kam. Der Tagesspiegel 22.12.2016. www.tagesspiegel.de/kultur/comics/ akira-dragon-ball-und-sailor-moonwie-der-manga-nach-deutschland$\mathrm{kam} / 14450220 . \mathrm{html}$.

VALTA, Reijo 2013. Quid? Asterix-latinan alkeita. Oulu: Osuuskunta Jyväs-Ainola.

VESTERINEN, Mika et al. 2007. Achtung! Korkkarisaksan sanakirja. Helsinki: Egmont.

ZEISBERGER, Ingold o. J. Weyarn in Bayern. Regionale Comics. Literaturportal Bayern. https://www.literaturportal-bayern.de. 